\title{
Composição e similaridade florística entre duas áreas de Floresta Atlântica Montana, São Paulo, Brasil
}

\author{
Maíra de Campos Gorgulho Padgurschi ${ }^{1,2}$, Larissa de Souza Pereira', \\ Jorge Yoshio Tamashiro ${ }^{1} \&$ Carlos Alfredo Joly ${ }^{1}$ \\ ${ }^{1}$ Departamento de Biologia Vegetal, Instituto de Biologia, Universidade Estadual de Campinas - \\ UNICAMP, CP 6109, CEP 13083-970, Campinas, SP, Brasil \\ ${ }^{2}$ Autor para correspondência: Maíra de Campos Gorgulho Padgurschi, e-mail: mairapad@gmail.com
}

PADGURSCHI, M.C.G., PEREIRA, L.P., TAMASHIRO, J.Y. \& JOLY, C.A. Floristic composition and similaritie between areas of Montane Atlantic Rainforest, São Paulo, Brazil. Biota Neotrop. 11(2): http:// www.biotaneotropica.org.br/v11n2/en/abstract?article+bn02811022011

\begin{abstract}
The study was conducted in two areas of Montana Atlantic Rainforest at Núcleo Santa Virgínia, Serra do Mar State Park, Brazil. The aim was to investigate structural and floristic composition of each area and the differences between them, knowing that one has not been disturbed recently and the other was subjected to selective logging until 1970, as reported by local people. We installed two 1 ha (PLOT K and PLOT N), approximately $4 \mathrm{~km}$ away from each other, and within this plots all individuals with $\mathrm{DBH} \geq 4.8 \mathrm{~cm}$ were recorded. Considering the two plots we sampled 3,503 individuals (2,269 trees $-64.7 \%$; 860 palms $-24.5 \%$; and 159 ferns $-4.5 \%)$, distributed in 265 species and 51 families. The rest ( 215 individuals) was dead. Among the most abundant families (Arecaceae, Myrtaceae, Lauraceae, Cyatheaceae) Monimiaceae is the only one classified as typical of the Montane Ombrophylus Dense Atlantic Forest. Euterpe edulis Mart. (Arecaceae) is the dominant species in PLOT K (old), where we recorded 1,852 individuals, 189 species and 43 families, with Myrtaceae (48), Lauraceae (26) and Monimiaceae (13) presenting the higher number of species. It is important to mention that clumps of a native bamboo (Merostachys neesii Ruprecht, Poaceae) are present in 93 of the 100 subparcels of PLOT K, summing up 3,813 culms. In contrast, in PLOT N (secondary) where palm heart (Euterpe edulis) is also the dominant species but bamboos are not so conspicuous, we recorded 1436 individuals, 149 species and 40 families, with Myrtaceae (27), Lauraceae (15) and Fabaceae (eight) being the ones with higher number of species. In the plot of secondary forest $(\mathrm{N})$ Shannon's diversity index $\left(\mathrm{H}^{\prime}=4.05\right)$ and the eveness index $\left(\mathrm{J}{ }^{\prime}=0.8\right)$ are higher than those recorded in the old plot of forest $(\mathrm{K})$ where $\mathrm{H}^{\prime}=3,72$ nats.ind $^{-1}$ and $\mathrm{J}^{\prime}=0.7$. Plots $\mathrm{K}$ and $\mathrm{N}$ have a low similarity (Jaccard index $\left.\mathrm{C}_{\mathrm{J}}=0,3\right)$, with only 94 species $(34,47 \%)$ in common, and $102(38,5 \%)$ occurring exclusively in PLOT K. However, the maximum estimate of species expected at the point of rarefaction of PLOT N (IC 95\% - 158.54) overlaps with the minimum estimate of species at the same point of PLOT K $(95 \%$ - 157.12), showing that the number of species of both areas would be equivalent in the number of 1,420 individuals. Although the largest tree sampled was found in PLOT K, where forest stratification is more evident, there is no significant difference between the sums of basal area of living individuals. Considering the disturbance history of the region, the results suggest that forest structure recovery may occur within 25 years but, as shown by the total number of species and by the diversity parameters determined, species richness does not recover within this time frame.
\end{abstract}

Keywords: tree species diversity, forest structure, pristine and secondary forest, Serra do Mar State Park, BIOTA Functional Gradient Project.

PADGURSCHI, M.C.G., PEREIRA, L.P., TAMASHIRO, J.Y. \& JOLY, C.A. Composição e similaridade florística entre duas áreas de Floresta Atlântica Montana, São Paulo, Brasil. Biota Neotrop. 11(2): http:// www.biotaneotropica.org.br/v11n2/pt/abstract?article+bn02811022011

Resumo: O trabalho foi desenvolvido em duas parcelas de 1 ha de Floresta Ombrófila Densa Montana do Núcleo Santa Virgínia, Parque Estadual da Serra do Mar, São Luiz do Paraitinga/SP, Brasil. Além de determinar a estrutura e a composição florística do componente arbóreo de cada parcela, o trabalho teve como objetivo comparar uma área (PLOT N) onde, segundo relatos de antigos moradores da região, houve corte seletivo de madeira até meados da década de 70 do século passado, com outra área (PLOT K) sem histórico de perturbação antrópica recente. As duas parcelas, que distam entre si cerca de $4 \mathrm{~km}$, foram subdivididas em 100 subparcelas de $10 \times 10 \mathrm{~m}$ e todos os indivíduos com DAP $\geq 4,8 \mathrm{~cm}$ foram marcados, mapeados, medidos e identificados. Ao todo foram amostrados 3.503 indivíduos, sendo 2.269 árvores $(64,7 \%), 860$ palmeiras $(24,5 \%)$ e $159(4,5 \%)$ fetos arborescentes, distribuídos em 265 espécies e 51 famílias. O restante dos indivíduos (215) estava morto. Dentre as famílias mais abundantes (Arecaceae, Myrtaceae, Lauraceae, Cyatheaceae), Monimiaceae é a única considerada típica da fitofisionomia Montana da Floresta Ombrófila Densa Atlântica. Euterpe edulis Mart. (Arecaceae) é a espécie dominante no PLOT K (pristina), onde foram registrados 1.852 indivíduos, distribuídos em 189 espécies e 43 famílias, sendo Myrtaceae (48), Lauraceae (26) e Monimiaceae (13) as que apresentaram a 
maior diversidade de espécies. É importante mencionar que moitas de bambu nativo (Merostachys neesii Ruprecht, Poaceae) estão presentes em 93 das 100 subparcelas desse plot, totalizando 3.813 colmos. Em contraste, no PLOT N, em que Euterpe edulis também é a espécie dominante, mas os bambus não estão tão presentes, foram identificados 1.436 indivíduos, distribuídos em 149 espécies e 40 famílias, com destaque para Myrtaceae (27), Lauraceae (15) e Fabaceae (oito) em termos de número de espécies. Na área de floresta secundária (PLOT N) o índice de diversidade de Shannon $\left(\mathrm{H}^{\prime}=4,05\right)$ e o índice de equidade $(\mathrm{J}$ ' $=0,8)$ são mais altos do que os valores encontrados na área que não sofreu corte seletivo (PLOT K) onde $\mathrm{H}^{\prime}=3,72$ nats.ind $^{-1} \mathrm{e} \mathrm{J}^{\prime}=0,7$. No entanto, a estimativa do número máximo de espécies esperado no ponto de rarefação do PLOT N (IC\% 95-158,54) se sobrepõe parcialmente à estimativa do número mínimo de espécies do PLOT K $(95 \%$ - 157,12), mostrando que o número de espécies de ambas as áreas se equivaleriam em 1420 indivíduos. Embora a maior árvore amostrada tenha sido encontrada no PLOT K, no qual os estratos da floresta são mais evidentes, não há diferença significativa entre as somas de área basal de indivíduos vivos das duas parcelas. Considerando o histórico de perturbação da região, os resultados sugerem que a recuperação da estrutura da floresta pode ocorrer dentro de 25 anos, mas, como mostrado pelo número total de espécies e pelo índice H' do PLOT K, este período é insuficiente para recuperação da diversidade de espécies arbóreas características da Floresta Ombrófila Densa Atlântica antiga.

Palavras-chave: diversidade de espécies arbóreas, estrutura florestal, floresta primária e secundária, Parque Estadual da Serra do Mar, Projeto BIOTA Gradiente Funcional.

\section{Introdução}

A Mata Atlântica (MA) sensu lato (Oliveira-Filho \& Fontes 2000) é a segunda maior floresta tropical do continente americano (Tabarelli et al. 2005). É classificada como um hotspot de biodiversidade (Myers et al 2000) e está entre as florestas mais ameaçadas do mundo, pois grande parte de sua área coincide com regiões populosas do Brasil (Oliveira-Filho \& Fontes 2000) onde, nas décadas de 50 e 60, cidades como Rio de Janeiro e São Paulo registraram altas taxas de crescimento gerando perdas e fragmentação do bioma (Ghazoul \& Sheil 2010). A agricultura e o comércio madeireiro estão entre os principais processos antrópicos que reduziram a MA (Dean 2002, Cesco \& Cabral 2008, Ghazoul \& Sheil 2010) para 11,4 a 16\% de sua cobertura original (Ribeiro et al. 2009). As paisagens que constituem a MA estão fragmentadas em áreas de diferentes tamanhos ao longo de sua distribuição (Ribeiro et al. 2009). Esta fragmentação somada à baixa disponibilidade de áreas primárias para a conservação da biodiversidade tem feito das florestas secundárias prioritárias para a conservação (Brearley et al. 2004, Liebsch et al. 2007). Segundo Hirota (2003), parte desses remanescentes está no estado de São Paulo, onde está também a maior área de proteção integral da MA: o Parque Estadual da Serra do Mar (PESM) (Instituto... 2010).

Com 315 mil ha, o PESM apresenta uma extensa área coberta por Floresta Ombrófila Densa e conta com oito núcleos administrativos, dentre os quais Santa Virgínia (NSV), local do presente estudo. O NSV está localizado a nordeste do PESM, em uma região importante do ponto de vista ecológico, já que somada às áreas do Parque Nacional da Serra da Bocaina e da Área de Proteção Ambiental de Cairuçu (ambos ao sul do estado do Rio de Janeiro) formam um contínuo de 120 mil ha de MA (Lacerda 2001). Entretanto, antes do estabelecimento do PESM, boa parte da região do NSV era composta por fazendas nas quais uma das principais atividades era a exploração madeireira (Aguirre 2008). Em entrevista, antigos funcionários da fazenda antecessora à implantação do NSV relataram o corte seletivo em algumas áreas de floresta. Espécies, como Chrysophyllum viride Mart. \& Eichler (Sapotaceae), eram retiradas para venda na indústria de carretéis (L. S. Pereira, dados não publicados) ou transformadas em pranchas para a indústria madeireira. Grande quantidade de taquaras (Merostachys neesii Ruprecht, Poaceae) também foi retirada para utilização na fabricação de cestos e balaios comercializados para o transporte de alimentos entre o interior e o litoral (Padgurschi 2010). A implantação do NSV, a partir da década de 80, cessou tais atividades e atualmente uma parte da floresta, que inclui a área perturbada, está incluída na zona intangível prevista no plano de manejo do PESM, onde somente pesquisas científicas são permitidas (Instituto... 2010).

Uma floresta é considerada, pois, secundária quando há supressão seletiva da vegetação e posterior abandono da atividade, deixando a região em processo natural de regeneração (Ghazoul \& Sheil 2010). Desde que próximas a matrizes florestais maduras e sem degradação do solo, florestas secundárias podem recuperar rapidamente (cerca de 30 anos) sua estrutura florestal, como área basal, densidade e riqueza (Brown \& Lugo 1990, Liebsch et al. 2007). Por outro lado, a semelhança na composição de espécies vegetais com áreas mais antigas será um processo mais lento, particularmente devido ao crescimento lento das árvores emergentes (Finegan 1996, Guariguata \& Ostertag 2001). A partir de diversos trabalhos realizados em diferentes áreas de floresta secundária neotropical, Guariguata \& Ostertag (2001) sugeriram uma sequência de eventos e processos que podem ocorrer nessas áreas logo após seu abandono até o retorno à composição de espécies similar às florestas menos perturbadas. Devido à grande extensão de florestas secundárias no neotrópico (Brown \& Lugo 1990) e, particularmente, na MA do sudeste do Brasil (Alves \& Metzger 2006), uma importante abordagem é o estado de conservação dessas florestas.

O presente estudo teve como objetivo verificar se há diferença na estrutura e composição florística entre duas áreas com diferentes históricos de uso. As seguintes questões nortearam a pesquisa: i) há diferença de estrutura arbórea entre as áreas? e ii) há diferenças na densidade de espécies e composição florística?

\section{Material e Métodos}

\section{1. Área de estudo}

OnúcleoSantaVirginia/PESM $\left(23^{\circ} 17^{\prime}\right.$ a $23^{\circ} 24^{\prime}$ S ; $45^{\circ} 03^{\prime}$ a $\left.45^{\circ} 11^{\prime} \mathrm{O}\right)$ tem sua maior área $(7.557 \mathrm{ha} / 44,5 \%)$ localizada no município de São Luiz do Paraitinga, Vale do Paraíba, São Paulo. Nessa região da Serra do Mar o relevo apresenta fortes declividades (24 a $37^{\circ}$ ) (Tabarelli et al. 1994) e o clima regional é do tipo Cwa na classificação de Köeppen (1948), com pluviosidade média anual de $2.500 \mathrm{~mm}$ (média entre 2008 e 2010, H. R. Rocha dados não publicados). O NSV apresenta-se como um mosaico composto por florestas secundárias, pastagens, plantio de Eucalyptus e floresta primária (Tabarelli et al. 1993). O estudo foi conduzido em 2 ha (Parcelas K \& N) de Floresta Ombrófila Densa Montana/NSV, ambos parte das 14 
parcelas permanentes do Projeto Temático Biota-Gradiente Funcional. As áreas localizam-se na região considerada zona intangível pelo plano de manejo do PESM (Instituto... 2010) e distam apenas 4 km entre si, e suas altitudes variam de 995 a 1093 m (Joly et al. 2011). Segundo entrevistas feitas com antigos moradores da região, o local de implantação da parcela $\mathrm{N}$ sofreu corte seletivo de madeira e retirada de taquaras por pelo menos 15 anos até meados da década de 70 do século passado. Este tipo de perturbação cessou quando a área foi anexada ao Parque Estadual da Serra do Mar. Portanto, trata-se de uma floresta secundária que está em processo de regeneração natural por, pelo menos, 25 anos.

O solo das áreas é muito semelhante. Apresenta textura franco-argilo-arenosa com $\mathrm{pH}$ muito baixo nos primeiros $10 \mathrm{~cm}$ (3,5 $\left.\mathrm{CaCl}_{2}\right)$ e baixa fertilidade, com elevados níveis de alumínio em todos os perfis (0 a $100 \mathrm{~cm}$ de profundidade) (Martins, 2010). Seguindo a classificação dada por Veloso et al. (1991), a região pertence ao grupo de formação Floresta Montana, que abaixo dos $23^{\circ}$ de latitude no estado de São Paulo ocorre entre 500 e 1500 m de altitude. Este grupo é caracterizado pela presença de neblina na maior parte do dia, raridade de trepadeiras lenhosas e abundância de epífitas, samambaias e taquaras (bamboo), componentes típicos de florestas montanas tropicais mais baixas (lower tropical mountains) (Ghazoul \& Sheil 2010).

\section{Levantamento florístico}

O estabelecimento das parcelas estudadas foi realizado de acordo com o protocolo de campo estabelecido por Joly et al. (2011). Cada parcela de 1 ha foi subdividida em 100 partes de $10 \times 10 \mathrm{~m}$ e todos os indivíduos (inclusive fetos arborescentes, palmeiras e mortos) com DAP $\geq 4,8 \mathrm{~cm}$ (PAP $\geq 15 \mathrm{~cm}$ ) foram marcados com placas de alumínio.

Para a coleta botânica, se o material estivesse fértil (com flores e/ou frutos) eram coletados cinco ramos, caso contrário apenas três (Fidalgo \& Bononi 1984) e a herborização seguiu as recomendações de Mori et al. (1989). As identificações foram feitas com a literatura pertinente, comparações com as coleções dos Herbários UEC e IAC e consultas aos especialistas. As espécies foram distribuídas por famílias reconhecidas pelo Angiosperm Phylogeny Group II (APG 2003) e as abreviações dos nomes dos autores foram realizadas segundo Brummitt \& Powell (1992).

\section{Estrutura da floresta}

A Fitossociologia refere-se ao estudo quantitativo da composição, estrutura, funcionamento, dinâmica, história, distribuição e relação ambiental da comunidade vegetal (Martins 1989). Neste trabalho os seguintes parâmetros foram utilizados (Martins 1991): densidade, frequência e dominância relativa, além do valor de importância e de cobertura (VI e VC, respectivamente) para cada família e espécie (Müeller-Dombois \& Ellenberg 1974). Os cálculos foram realizados com o auxílio do programa FITOPAC (Shepherd 2008).

Para avaliar as diferenças estruturais entre as parcelas, foram utilizados os descritores: área basal, densidade absoluta de árvores e altura das árvores (para verificar a estratificação do dossel). As diferenças para os dois primeiros foram avaliadas realizando-se o teste $t$ de Student (Zar 1999) com nível de relevância de 95\%. Os testes foram realizados com o programa Biostat 5.0 (Ayres et al., 2007). Para verificar a estratificação foi realizada a distribuição das frequências de altura em seis diferentes classes de tamanho.

Como o número total de indivíduos foi diferente entre as parcelas, a comparação entre os valores de densidade de espécies (Densidade de espécies é definida por Magurran (2004) como o número de espécies presentes em determinada área. Em muitos trabalhos encontra-se o termo riqueza, porém este é abordado aqui como parte da heterogeneidade) foi realizada em um ponto padronizado (rarefação), definido com base no número de indivíduos da menor amostra (Parcela N). Nessa etapa utilizou-se o número esperado de espécies por subparcela calculado segundo a fórmula analítica "Mao Tau" (Colwell et al. 2004, Mao \& Colwell 2005). A análise foi realizada no programa EstimateS versão 8.2 (Colwell 2009), que além da densidade de espécies ("Sobs Mao Tau”) forneceu os intervalos de confiança de 95\% (IC 95\%). As curvas de acumulação de espécies, reescalonando o eixo $\times$ pelo número de indivíduos conforme recomendado por Gotelli \& Colwell (2001), foi realizada no programa Excel. Para avaliação da diversidade entre as áreas (ou heterogeneidade - Magurran (2004)), foi calculado o índice de diversidade de Shannon-Weiner (H') e equabilidade de Pielou (J') (Brower \& Zar 1984) com o auxílio do programa FITOPAC (Shepherd 2008). Como índice não mede a diversidade em si (Jost 2010), foi realizada a conversão deste em número efetivo de espécies, que é o número necessário de espécies igualmente distribuídas para resultar em um valor particular do índice utilizado (Jost 2006). A similaridade entre as áreas foi avaliada segundo o coeficiente de Jaccard $\left(\mathrm{C}_{\mathrm{j}}\right)$, pois este não considera a frequência das espécies e deve-se, pois, usá-lo quando se deseja pesar as espécies de maneira igualitária (Krebs 1999).

\section{Resultados}

Considerando as duas áreas de estudo, foram incluídos 3.503 indivíduos dos quais $215(7,1 \%)$ estavam mortos. Do restante, $3.129(95,2 \%)$ são árvores e $159(4,8 \%)$ são fetos arborescentes todos da família Cyatheaceae. Foram encontradas 265 espécies e 51 famílias botânicas. Alguns indivíduos (31) estão como "indeterminados", pois não foram coletados devido à altura de seus ramos. Foram identificados 37 indivíduos ao nível de família e 108 apenas ao nível de gênero (Tabelas 1 e 2).

Somado os hectares, Arecaceae foi a família mais abundante (860 ind.) representada por apenas uma espécie, Euterpe edulis Mart., popularmente conhecida como palmito "Jussara". Em seguida estão Myrtaceae, Lauraceae e Monimiaceae com 561, 255 e 215 indivíduos, respectivamente (Tabelas 1 e 2). As famílias com maior densidade de espécies foram Myrtaceae (57), Lauraceae (33), Fabaceae e Monimiaceae (15 espécies cada). No entanto, metade das famílias (26) apresentou uma ou duas espécies apenas (Figura 1). Excetuando-se E. edulis, as espécies mais abundantes foram Myrcia spectabilis DC. (102), Licania hoehnei Pilg. (95), Guapira opposita (Vell.) Reitz e Bathysa australis (A. St.-Hil.) Benth. \& Hook. (80 cada), Ocotea catharinensis Mez (79) e Chrysophyllum viride Mart. \& Eichler (72). No outro extremo estão 81 espécies que foram representadas por um único indivíduo (Tabelas 1 e 2). Juntas, as duas áreas apresentaram índice de Shannon-Weiner de H' = 4,08 nats.ind ${ }^{-1}$ e equabilidade de $\mathrm{J}^{\prime}=0,73$ (Tabela 3$)$.

O diâmetro (DAP) máximo obtido foi $108,86 \mathrm{~cm}$ e a altura máxima foi $30 \mathrm{~m}$, ambos na comunidade florestal $\mathrm{K}$, onde se observou também dois estratos a mais na floresta (árvores $<5 \mathrm{~m} \mathrm{e} \geq 25,1 \mathrm{~m}$ ) (Figura 2). Porém, nesta área a altura média foi menor (9,1 m \pm 4 d.p) quando comparada à comunidade $\mathrm{N}(11,7 \mathrm{~m} \pm 3,9 \mathrm{~d}$.p). O DAP médio $(12,74 \mathrm{~cm})$ também foi significativamente menor quando comparado à parcela $\mathrm{N}(14,29 \mathrm{~cm})(\mathrm{p}<0,001 ; \mathrm{t}=3,54 ; \mathrm{gl}=198)$. Já para a soma da área basal dos indivíduos vivos a parcela $\mathrm{K}$ apresentou valor superior à parcela $\mathrm{N}\left(40,20 \mathrm{~m}^{2} \cdot \mathrm{ha}^{-1} \mathrm{e} 37,47 \mathrm{~m}^{2} \cdot \mathrm{ha}^{-1}\right.$ respectivamente), mas o valor não foi significativamente distinto $(\mathrm{p}>0,01 ; \mathrm{t}=0,88$; $\mathrm{gl}=198)($ Tabela 3$)$.

A parcela K obteve densidade absoluta maior do que a N $(1.852 \mathrm{e}$ 1.436, respectivamente $\mathrm{p}<0,0001)$. A comparação de densidade de espécies entre as áreas foi realizada, pois, no ponto de rarefação 
Padgurschi, M.C.G. et al.

Tabela 1. Fitossociologia da Parcela K (antiga). As espécies estão classificadas pelo valor decrescente de V.I. n.i. - número de indivíduos; n.a. - número de amostras em que a espécie ocorreu; De.R. - densidade relativa; Do.R. - dominância relativa; Fr.R.- frequência relativa; V.I. - valor de importância; V.C. valor de cobertura.

Table 1. Phytosociology of Plot K (old). Species are ranked by decreasing value of V.I. n.i. - number of individuals; n.a. - number of samples where the species occurred; De.R. - relative density; Do.R. - relative dominance; Fr.R. - relative frequency; V.I. - value of importance; V.C. - value coverage.

\begin{tabular}{|c|c|c|c|c|c|c|c|c|}
\hline Espécie & Família & n.i. & n.a. & De.R & Do.R & Fr.R & V.I. & V.C. \\
\hline Euterpe edulis Mart. & Arecaceae & 562 & 93 & 30,35 & 13,1 & 8,35 & 51,8 & 43,45 \\
\hline Chrysophyllum viride Mart. \& Eichler & Sapotaceae & 52 & 35 & 2,81 & 16,5 & 3,14 & 22,45 & 19,31 \\
\hline Licania hoehnei Pilg. & Chrysobalanaceae & 53 & 30 & 2,86 & 6 & 2,69 & 11,55 & 8,86 \\
\hline Guapira opposita (Vell.) Reitz & Nyctaginaceae & 77 & 32 & 4,16 & 3,99 & 2,87 & 11,02 & 8,15 \\
\hline Ocotea catharinensis $\mathrm{Mez}$ & Lauraceae & 42 & 31 & 2,27 & 5,85 & 2,78 & 10,9 & 8,12 \\
\hline Myrcia spectabilis DC. & Myrtaceae & 65 & 46 & 3,51 & 2,68 & 4,13 & 10,32 & 6,19 \\
\hline Bathysa australis (A.St.-Hil.) Benth. \& Hook. & Rubiaceae & 54 & 31 & 2,92 & 2,01 & 2,78 & 7,71 & 4,92 \\
\hline Pouteria caimito (Ruiz \& Pav.) Radlk. & Sapotaceae & 45 & 33 & 2,43 & 1,29 & 2,96 & 6,68 & 3,72 \\
\hline Mollinedia argyrogyna Perkins & Monimiaceae & 32 & 25 & 1,73 & 1,44 & 2,24 & 5,41 & 3,17 \\
\hline Couepia venosa Prance & Chrysobalanaceae & 19 & 17 & 1,03 & 2,76 & 1,53 & 5,31 & 3,78 \\
\hline Alchornea glandulosa Poepp. & Euphorbiaceae & 11 & 11 & 0,59 & 3,44 & 0,99 & 5,02 & 4,03 \\
\hline Cyathea dichromatolepis (Fée) Domin & Cyatheaceae & 33 & 23 & 1,78 & 0,31 & 2,06 & 4,16 & 2,1 \\
\hline Ocotea dispersa (Nees \& Mart.) Mez & Lauraceae & 23 & 20 & 1,24 & 0,55 & 1,8 & 3,58 & 1,79 \\
\hline Mollinedia salicifolia Perkins & Monimiaceae & 23 & 21 & 1,24 & 0,4 & 1,89 & 3,52 & 1,64 \\
\hline Amaioua intermedia Mart. & Rubiaceae & 18 & 16 & 0,97 & 0,93 & 1,44 & 3,34 & 1,9 \\
\hline Schefflera angustissima (Marchal) Frodin & Araliaceae & 11 & 11 & 0,59 & 1,43 & 0,99 & 3,01 & 2,02 \\
\hline Calyptranthes grandifolia O.Berg & Myrtaceae & 18 & 16 & 0,97 & 0,51 & 1,44 & 2,92 & 1,48 \\
\hline Eugenia prasina O.Berg & Myrtaceae & 23 & 16 & 1,24 & 0,21 & 1,44 & 2,89 & 1,45 \\
\hline Cyathea phalerata Mart. & Cyatheaceae & 20 & 16 & 1,08 & 0,34 & 1,44 & 2,86 & 1,42 \\
\hline Miconia cabucu Hoehne & Melastomataceae & 11 & 10 & 0,59 & 1,26 & 0,9 & 2,76 & 1,86 \\
\hline Marlierea excoriata Mart. & Myrtaceae & 16 & 15 & 0,86 & 0,54 & 1,35 & 2,75 & 1,4 \\
\hline Calyptranthes cf. lucida Mart. ex DC. & Myrtaceae & 16 & 10 & 0,86 & 0,84 & 0,9 & 2,6 & 1,7 \\
\hline Cryptocarya saligna $\mathrm{Mez}$ & Lauraceae & 13 & 12 & 0,7 & 0,79 & 1,08 & 2,57 & 1,5 \\
\hline Calyptranthes lucida Mart. ex DC. & Myrtaceae & 17 & 15 & 0,92 & 0,29 & 1,35 & 2,55 & 1,21 \\
\hline Alsophila sternbergii (Sternb.) D.S.Conant & Cyatheaceae & 25 & 5 & 1,35 & 0,7 & 0,45 & 2,5 & 2,05 \\
\hline Cordia trichoclada DC. & Boraginaceae & 15 & 13 & 0,81 & 0,48 & 1,17 & 2,46 & 1,29 \\
\hline Aiouea acarodomatifera Kosterm. & Lauraceae & 6 & 5 & 0,32 & 1,65 & 0,45 & 2,42 & 1,97 \\
\hline Coussapoa microcarpa (Schott) Rizzini & Urticaceae & 7 & 7 & 0,38 & 1,4 & 0,63 & 2,41 & 1,78 \\
\hline Myrciaria floribunda (H.West ex Willd.) O.Berg & Myrtaceae & 16 & 13 & 0,86 & 0,34 & 1,17 & 2,38 & 1,21 \\
\hline Myrtaceae sp. 2 & Myrtaceae & 3 & 3 & 0,16 & 1,92 & 0,27 & 2,35 & 2,08 \\
\hline Hirtella hebeclada Moric. ex DC. & Chrysobalanaceae & 14 & 10 & 0,76 & 0,67 & 0,9 & 2,33 & 1,43 \\
\hline Cabralea canjerana subsp. canjerana (Vell.) Mart. & Meliaceae & 5 & 5 & 0,27 & 1,56 & 0,45 & 2,28 & 1,83 \\
\hline Cryptocarya cf. moschata Nees \& Mart. & Lauraceae & 7 & 7 & 0,38 & 1,25 & 0,63 & 2,26 & 1,63 \\
\hline Guatteria australis A.St.-Hil. & Annonaceae & 14 & 13 & 0,76 & 0,22 & 1,17 & 2,14 & 0,97 \\
\hline Chionanthus crassifolius var. crassifolius (Mart.) P.S.Green & Oleaceae & 13 & 13 & 0,7 & 0,24 & 1,17 & 2,11 & 0,94 \\
\hline Jacaranda montana Morawetz & Bignoniaceae & 9 & 8 & 0,49 & 0,77 & 0,72 & 1,98 & 1,26 \\
\hline Mollinedia schottiana (Spreng.) Perkins & Monimiaceae & 14 & 12 & 0,76 & 0,15 & 1,08 & 1,98 & 0,9 \\
\hline Matayba guianensis Aubl. & Sapindaceae & 13 & 10 & 0,7 & 0,26 & 0,9 & 1,86 & 0,96 \\
\hline Rapanea hermogenesii Jung-Mend. \& Bernacci & Myrsinaceae & 7 & 6 & 0,38 & 0,84 & 0,54 & 1,76 & 1,22 \\
\hline Schefflera calva (Cham.) Frodin \& Fiaschi & Araliaceae & 11 & 10 & 0,59 & 0,26 & 0,9 & 1,75 & 0,85 \\
\hline Cyathea delgadii Sternb. & Cyatheaceae & 10 & 9 & 0,54 & 0,22 & 0,81 & 1,57 & 0,76 \\
\hline Calyptranthes strigipes O.Berg & Myrtaceae & 8 & 8 & 0,43 & 0,33 & 0,72 & 1,48 & 0,76 \\
\hline Drimys brasiliensis Miers & Winteraceae & 5 & 5 & 0,27 & 0,71 & 0,45 & 1,43 & 0,98 \\
\hline Miconia brasiliensis (Spreng.) Triana & Melastomataceae & 10 & 8 & 0,54 & 0,1 & 0,72 & 1,35 & 0,64 \\
\hline Myrcia tijucensis Kiaersk. & Myrtaceae & 8 & 8 & 0,43 & 0,13 & 0,72 & 1,28 & 0,56 \\
\hline Psychotria vellosiana Benth. & Rubiaceae & 8 & 7 & 0,43 & 0,21 & 0,63 & 1,27 & 0,64 \\
\hline Guarea macrophylla subsp. tuberculata (Vell.) T.D.Penn. & Meliaceae & 8 & 8 & 0,43 & 0,1 & 0,72 & 1,26 & 0,54 \\
\hline
\end{tabular}

As espécies em cinza são consideradas exclusivas da área $\mathrm{K}$.

Species in gray color are considered unique to the area $\mathrm{K}$. 
Tabela 1. Continuação...

\begin{tabular}{|c|c|c|c|c|c|c|c|c|}
\hline Espécie & Família & n.i. & n.a. & De.R & Do.R & Fr.R & V.I. & V.C. \\
\hline Roupala brasiliensis Klotzsch & Proteaceae & 9 & 6 & 0,49 & 0,24 & 0,54 & 1,26 & 0,73 \\
\hline Marlierea cf. excoriata Mart. & Myrtaceae & 8 & 8 & 0,43 & 0,09 & 0,72 & 1,24 & 0,52 \\
\hline Eugenia sp. 2 & Myrtaceae & 4 & 4 & 0,22 & 0,58 & 0,36 & 1,15 & 0,79 \\
\hline Ocotea aciphylla (Nees \& Mart.) Mez & Lauraceae & 6 & 6 & 0,32 & 0,25 & 0,54 & 1,11 & 0,57 \\
\hline Eugenia sp. 1 & Myrtaceae & 8 & 6 & 0,43 & 0,12 & 0,54 & 1,09 & 0,55 \\
\hline Ocotea daphnifolia (Meisn.) Mez & Lauraceae & 7 & 6 & 0,38 & 0,18 & 0,54 & 1,09 & 0,56 \\
\hline Henriettella glabra (Vell.) Cogn. & Melastomataceae & 4 & 4 & 0,22 & 0,5 & 0,36 & 1,08 & 0,72 \\
\hline Mollinedia aff. salicifolia Perkins & Monimiaceae & 8 & 6 & 0,43 & 0,11 & 0,54 & 1,08 & 0,55 \\
\hline Croton macrobothrys Baill. & Euphorbiaceae & 3 & 3 & 0,16 & 0,63 & 0,27 & 1,06 & 0,79 \\
\hline Eugenia oblongata O.Berg & Myrtaceae & 6 & 6 & 0,32 & 0,2 & 0,54 & 1,06 & 0,52 \\
\hline Matayba intermedia Radlk. & Sapindaceae & 4 & 3 & 0,22 & 0,57 & 0,27 & 1,05 & 0,78 \\
\hline Heisteria silvianii Schwacke & Olacaceae & 3 & 3 & 0,16 & 0,56 & 0,27 & 1 & 0,73 \\
\hline Eugenia cf. prasina O.Berg & Myrtaceae & 6 & 6 & 0,32 & 0,12 & 0,54 & 0,98 & 0,44 \\
\hline Cecropia glaziovii Snethl. & Urticaceae & 5 & 4 & 0,27 & 0,34 & 0,36 & 0,97 & 0,61 \\
\hline Sloanea hirsuta (Schott) Planch. ex Benth. & Elaeocarpaceae & 5 & 5 & 0,27 & 0,25 & 0,45 & 0,97 & 0,52 \\
\hline Calyptranthes sp. 1 & Myrtaceae & 6 & 6 & 0,32 & 0,07 & 0,54 & 0,93 & 0,39 \\
\hline Calyptranthes cf. grandifolia O.Berg & Myrtaceae & 6 & 6 & 0,32 & 0,05 & 0,54 & 0,92 & 0,38 \\
\hline Calyptranthes rufa $\mathrm{O}$. Berg & Myrtaceae & 6 & 6 & 0,32 & 0,05 & 0,54 & 0,91 & 0,37 \\
\hline Rapanea gardneriana (A. DC.) Mez & Myrsinaceae & 5 & 5 & 0,27 & 0,19 & 0,45 & 0,91 & 0,46 \\
\hline Mollinedia blumenaviana Perkins & Monimiaceae & 7 & 5 & 0,38 & 0,05 & 0,45 & 0,88 & 0,43 \\
\hline Myrceugenia miersiana (Gardner) D.Legrand \& Kausel & Myrtaceae & 5 & 5 & 0,27 & 0,16 & 0,45 & 0,88 & 0,43 \\
\hline Myrcia cf. guianensis (Aubl.) DC. & Myrtaceae & 2 & 2 & 0,11 & 0,59 & 0,18 & 0,88 & 0,7 \\
\hline Tachigali sp. 1 & Fabaceae & 2 & 2 & 0,11 & 0,59 & 0,18 & 0,88 & 0,7 \\
\hline Lauraceae sp. 3 & Lauraceae & 1 & 1 & 0,05 & 0,72 & 0,09 & 0,87 & 0,78 \\
\hline Symplocos laxiflora Benth. & Symplocaceae & 6 & 5 & 0,32 & 0,09 & 0,45 & 0,86 & 0,41 \\
\hline Ocotea cf. lancifolia (Schott) Mez & Lauraceae & 6 & 5 & 0,32 & 0,07 & 0,45 & 0,85 & 0,4 \\
\hline Allophylus petiolulatus Radlk. & Sapindaceae & 5 & 5 & 0,27 & 0,1 & 0,45 & 0,82 & 0,37 \\
\hline Neomitranthes glomerata (D.Legrand) D.Legrand & Myrtaceae & 4 & 4 & 0,22 & 0,24 & 0,36 & 0,82 & 0,46 \\
\hline Chrysophyllum inornatum Mart. & Sapotaceae & 5 & 5 & 0,27 & 0,09 & 0,45 & 0,81 & 0,36 \\
\hline Stephanopodium sessile Rizzini & Dichapetalaceae & 4 & 3 & 0,22 & 0,33 & 0,27 & 0,81 & 0,54 \\
\hline Cupania vernalis Cambess. & Sapindaceae & 5 & 5 & 0,27 & 0,08 & 0,45 & 0,8 & 0,35 \\
\hline Daphnopsis schwackeana Taub. & Thymelaeaceae & 5 & 5 & 0,27 & 0,07 & 0,45 & 0,79 & 0,34 \\
\hline Rutaceae sp. 1 & Rutaceae & 4 & 4 & 0,22 & 0,22 & 0,36 & 0,79 & 0,43 \\
\hline Posoqueria latifolia (Rudge) Schult. & Rubiaceae & 5 & 5 & 0,27 & 0,06 & 0,45 & 0,78 & 0,33 \\
\hline Rudgea vellerea Müll.Arg. & Rubiaceae & 4 & 4 & 0,22 & 0,21 & 0,36 & 0,78 & 0,42 \\
\hline Aniba viridis $\mathrm{Mez}$ & Lauraceae & 5 & 5 & 0,27 & 0,04 & 0,45 & 0,76 & 0,31 \\
\hline Rapanea guianensis Aubl. & Myrsinaceae & 5 & 5 & 0,27 & 0,05 & 0,45 & 0,76 & 0,32 \\
\hline Sapium glandulosum (L.) Morong & Euphorbiaceae & 1 & 1 & 0,05 & 0,61 & 0,09 & 0,76 & 0,67 \\
\hline Cupania furfuracea Radlk. & Sapindaceae & 3 & 3 & 0,16 & 0,32 & 0,27 & 0,75 & 0,48 \\
\hline Dahlstedtia pinnata (Benth.) Malme & Fabaceae & 5 & 5 & 0,27 & 0,03 & 0,45 & 0,75 & 0,3 \\
\hline Inga lanceifolia Benth. & Fabaceae & 5 & 4 & 0,27 & 0,09 & 0,36 & 0,72 & 0,36 \\
\hline Marlierea silvatica (O.Berg) Kiaersk. & Myrtaceae & 3 & 3 & 0,16 & 0,27 & 0,27 & 0,7 & 0,43 \\
\hline Mollinedia boracensis Peixoto & Monimiaceae & 5 & 4 & 0,27 & 0,07 & 0,36 & 0,7 & 0,34 \\
\hline Ormosia minor Vogel & Fabaceae & 5 & 4 & 0,27 & 0,07 & 0,36 & 0,7 & 0,34 \\
\hline Stephanopodium blanchetianum Baill. & Dichapetalaceae & 3 & 3 & 0,16 & 0,27 & 0,27 & 0,7 & 0,43 \\
\hline Myrcia splendens (Sw.) DC. & Myrtaceae & 4 & 4 & 0,22 & 0,06 & 0,36 & 0,64 & 0,28 \\
\hline Clusia criuva var. parviflora Vesque & Clusiaceae & 1 & 1 & 0,05 & 0,48 & 0,09 & 0,63 & 0,54 \\
\hline Jacaratia spinosa (Aubl.) A.DC. & Caricaceae & 1 & 1 & 0,05 & 0,49 & 0,09 & 0,63 & 0,54 \\
\hline Ocotea silvestris Vattimo-Gil & Lauraceae & 3 & 3 & 0,16 & 0,2 & 0,27 & 0,63 & 0,36 \\
\hline Myrceugenia campestris (DC.) D.Legrand \& Kausel & Myrtaceae & 5 & 3 & 0,27 & 0,03 & 0,27 & 0,57 & 0,3 \\
\hline Tibouchina cf. arborea (Gardner) Cogn. & Melastomataceae & 2 & 2 & 0,11 & 0,28 & 0,18 & 0,57 & 0,39 \\
\hline
\end{tabular}

As espécies em cinza são consideradas exclusivas da área $\mathrm{K}$.

Species in gray color are considered unique to the area $\mathrm{K}$. 
Tabela 1. Continuação...

\begin{tabular}{|c|c|c|c|c|c|c|c|c|}
\hline Espécie & Família & n.i. & n.a. & De.R & Do.R & Fr.R & V.I. & V.C. \\
\hline Marlierea tomentosa Cambess. & Myrtaceae & 3 & 3 & 0,16 & 0,12 & 0,27 & 0,56 & 0,29 \\
\hline Mollinedia gilgiana Perkins & Monimiaceae & 4 & 3 & 0,22 & 0,08 & 0,27 & 0,56 & 0,29 \\
\hline Mollinedia engleriana Perkins & Monimiaceae & 3 & 3 & 0,16 & 0,09 & 0,27 & 0,53 & 0,26 \\
\hline Myrtaceae sp. 8 & Myrtaceae & 2 & 2 & 0,11 & 0,23 & 0,18 & 0,51 & 0,33 \\
\hline Ocotea puberula (Rich.) Nees & Lauraceae & 3 & 3 & 0,16 & 0,08 & 0,27 & 0,51 & 0,24 \\
\hline Garcinia gardneriana (Planch. \& Triana) Zappi & Clusiaceae & 3 & 3 & 0,16 & 0,04 & 0,27 & 0,47 & 0,2 \\
\hline Nectandra sp. 1 & Lauraceae & 1 & 1 & 0,05 & 0,33 & 0,09 & 0,47 & 0,38 \\
\hline Myrtaceae sp. 6 & Myrtaceae & 1 & 1 & 0,05 & 0,31 & 0,09 & 0,46 & 0,37 \\
\hline Tibouchina pulchra Cogn. & Melastomataceae & 2 & 2 & 0,11 & 0,17 & 0,18 & 0,46 & 0,28 \\
\hline Tachigali friburgensis (Harms) L.G.Silva \& H.C.Lima & Fabaceae & 2 & 2 & 0,11 & 0,16 & 0,18 & 0,45 & 0,27 \\
\hline Lauraceae sp. 4 & Lauraceae & 2 & 2 & 0,11 & 0,15 & 0,18 & 0,43 & 0,25 \\
\hline Ocotea cf. dispersa (Nees \& Mart.) Mez & Lauraceae & 1 & 1 & 0,05 & 0,29 & 0,09 & 0,43 & 0,34 \\
\hline Alsophila setosa Kaulf. & Cyatheaceae & 3 & 2 & 0,16 & 0,06 & 0,18 & 0,4 & 0,22 \\
\hline Cryptocarya aschersoniana $\mathrm{Mez}$ & Lauraceae & 2 & 2 & 0,11 & 0,11 & 0,18 & 0,4 & 0,22 \\
\hline Ocotea bicolor Vattimo-Gil & Lauraceae & 2 & 2 & 0,11 & 0,11 & 0,18 & 0,4 & 0,22 \\
\hline Nectandra membranacea (Sw.) Griseb. & Lauraceae & 2 & 2 & 0,11 & 0,09 & 0,18 & 0,38 & 0,2 \\
\hline Cordia taguahyensis Vell. & Boraginaceae & 3 & 2 & 0,16 & 0,03 & 0,18 & 0,37 & 0,19 \\
\hline Blepharocalyx salicifolius (Kunth) O. Berg & Myrtaceae & 2 & 2 & 0,11 & 0,07 & 0,18 & 0,36 & 0,18 \\
\hline Ouratea multiflora (Pohl) Engl. & Ochnaceae & 3 & 2 & 0,16 & 0,02 & 0,18 & 0,36 & 0,19 \\
\hline Lamanonia ternata Vell. & Cunoniaceae & 2 & 2 & 0,11 & 0,06 & 0,18 & 0,35 & 0,17 \\
\hline Beilschmiedia emarginata (Meisn.) Kosterm. & Lauraceae & 1 & 1 & 0,05 & 0,19 & 0,09 & 0,34 & 0,25 \\
\hline Ocotea laxa (Nees) Mez & Lauraceae & 1 & 1 & 0,05 & 0,19 & 0,09 & 0,33 & 0,24 \\
\hline Tachigali sp. 2 & Fabaceae & 2 & 2 & 0,11 & 0,04 & 0,18 & 0,33 & 0,15 \\
\hline Casearia obliqua Spreng. & Salicaceae & 1 & 1 & 0,05 & 0,18 & 0,09 & 0,32 & 0,23 \\
\hline Piptocarpha macropoda (DC.) Baker & Asteraceae & 1 & 1 & 0,05 & 0,17 & 0,09 & 0,32 & 0,23 \\
\hline Cordia sellowiana Cham. & Boraginaceae & 2 & 2 & 0,11 & 0,02 & 0,18 & 0,31 & 0,13 \\
\hline Cupania oblongifolia Mart. & Sapindaceae & 2 & 2 & 0,11 & 0,02 & 0,18 & 0,31 & 0,13 \\
\hline Myrcia amazonica DC. & Myrtaceae & 2 & 2 & 0,11 & 0,03 & 0,18 & 0,31 & 0,13 \\
\hline Myrtaceae sp. 9 & Myrtaceae & 2 & 2 & 0,11 & 0,02 & 0,18 & 0,31 & 0,13 \\
\hline Ocotea corymbosa (Meisn.) Mez & Lauraceae & 1 & 1 & 0,05 & 0,17 & 0,09 & 0,31 & 0,22 \\
\hline Ocotea teleiandra (Meisn.) Mez & Lauraceae & 2 & 2 & 0,11 & 0,03 & 0,18 & 0,31 & 0,14 \\
\hline Symplocos estrellensis Casar. & Symplocaceae & 2 & 2 & 0,11 & 0,02 & 0,18 & 0,31 & 0,13 \\
\hline Eugenia cf. multicostata D.Legrand & Myrtaceae & 1 & 1 & 0,05 & 0,15 & 0,09 & 0,3 & 0,21 \\
\hline Eugenia sp. 4 & Myrtaceae & 2 & 2 & 0,11 & 0,01 & 0,18 & 0,3 & 0,12 \\
\hline Byrsonima salzmanniana A. Juss. & Malpighiaceae & 1 & 1 & 0,05 & 0,15 & 0,09 & 0,29 & 0,2 \\
\hline Ilex paraguariensis A.St.-Hil. & Aquifoliaceae & 1 & 1 & 0,05 & 0,15 & 0,09 & 0,29 & 0,2 \\
\hline Myrtaceae sp. 5 & Myrtaceae & 1 & 1 & 0,05 & 0,14 & 0,09 & 0,28 & 0,19 \\
\hline Ocotea elegans $\mathrm{Mez}$ & Lauraceae & 2 & 1 & 0,11 & 0,07 & 0,09 & 0,27 & 0,18 \\
\hline Prunus myrtifolia (L.) Urb. & Rosaceae & 1 & 1 & 0,05 & 0,11 & 0,09 & 0,25 & 0,16 \\
\hline Eugenia burkartiana (D.Legrand) D.Legrand & Myrtaceae & 1 & 1 & 0,05 & 0,1 & 0,09 & 0,24 & 0,15 \\
\hline Ilex brevicuspis Reissek & Aquifoliaceae & 1 & 1 & 0,05 & 0,1 & 0,09 & 0,24 & 0,15 \\
\hline Eugenia copacabanensis Kiaersk. & Myrtaceae & 1 & 1 & 0,05 & 0,09 & 0,09 & 0,23 & 0,14 \\
\hline Myrtaceae sp. 7 & Myrtaceae & 1 & 1 & 0,05 & 0,09 & 0,09 & 0,23 & 0,14 \\
\hline Calyptranthes cf. strigipes O.Berg & Myrtaceae & 1 & 1 & 0,05 & 0,08 & 0,09 & 0,22 & 0,13 \\
\hline Myrtaceae sp. 4 & Myrtaceae & 1 & 1 & 0,05 & 0,08 & 0,09 & 0,22 & 0,13 \\
\hline Myrcia cf. amazonica DC. & Myrtaceae & 2 & 1 & 0,11 & 0,02 & 0,09 & 0,21 & 0,12 \\
\hline Ormosia sp. 1 & Fabaceae & 1 & 1 & 0,05 & 0,07 & 0,09 & 0,21 & 0,12 \\
\hline Lauraceae sp. 2 & Lauraceae & 1 & 1 & 0,05 & 0,06 & 0,09 & 0,2 & 0,11 \\
\hline Mollinedia sp. 2 & Monimiaceae & 1 & 1 & 0,05 & 0,06 & 0,09 & 0,2 & 0,11 \\
\hline Roupala consimilis Mez ex Taub. & Proteaceae & 1 & 1 & 0,05 & 0,06 & 0,09 & 0,2 & 0,11 \\
\hline Coussarea meridionalis var. porophylla (Vell.) M.Gomes & Rubiaceae & 1 & 1 & 0,05 & 0,05 & 0,09 & 0,19 & 0,1 \\
\hline
\end{tabular}

As espécies em cinza são consideradas exclusivas da área $\mathrm{K}$.

Species in gray color are considered unique to the area $\mathrm{K}$. 
Tabela 1. Continuação...

\begin{tabular}{|c|c|c|c|c|c|c|c|c|}
\hline Espécie & Família & n.i. & n.a. & De.R & Do.R & Fr.R & V.I. & V.C. \\
\hline Endlicheria paniculata (Spreng.) J.F.Macbr. & Lauraceae & 1 & 1 & 0,05 & 0,04 & 0,09 & 0,19 & 0,1 \\
\hline Eugenia mosenii (Kausel) Sobral & Myrtaceae & 1 & 1 & 0,05 & 0,03 & 0,09 & 0,18 & 0,09 \\
\hline Miconia cubatanensis Hoehne & Melastomataceae & 1 & 1 & 0,05 & 0,04 & 0,09 & 0,18 & 0,09 \\
\hline Roupala paulensis Sleumer & Proteaceae & 1 & 1 & 0,05 & 0,03 & 0,09 & 0,18 & 0,09 \\
\hline Casearia sylvestris $\mathrm{Sw}$. & Salicaceae & 1 & 1 & 0,05 & 0,03 & 0,09 & 0,17 & 0,08 \\
\hline Citronella paniculata (Mart.) R.A.Howard & Cardiopteridaceae & 1 & 1 & 0,05 & 0,03 & 0,09 & 0,17 & 0,08 \\
\hline Guapira hirsuta (Choisy) Lundell & Nyctaginaceae & 1 & 1 & 0,05 & 0,02 & 0,09 & 0,17 & 0,08 \\
\hline Phytolacaceae sp. 1 & Phytolacaceae & 1 & 1 & 0,05 & 0,03 & 0,09 & 0,17 & 0,08 \\
\hline Chrysophyllum flexuosum Mart. & Sapotaceae & 1 & 1 & 0,05 & 0,01 & 0,09 & 0,16 & 0,07 \\
\hline Conchocarpus fontanesianus (A. St.-Hil.) Kallunki \& Pirani & Rutaceae & 1 & 1 & 0,05 & 0,01 & 0,09 & 0,16 & 0,07 \\
\hline Coussarea sp. 1 & Rubiaceae & 1 & 1 & 0,05 & 0,02 & 0,09 & 0,16 & 0,07 \\
\hline Cryptocarya moschata Nees \& Mart. & Lauraceae & 1 & 1 & 0,05 & 0,01 & 0,09 & 0,16 & 0,07 \\
\hline Cupania zanthoxyloides Radlk. & Sapindaceae & 1 & 1 & 0,05 & 0,02 & 0,09 & 0,16 & 0,07 \\
\hline Ilex microdonta Reissek & Aquifoliaceae & 1 & 1 & 0,05 & 0,02 & 0,09 & 0,16 & 0,07 \\
\hline Marlierea sp. 1 & Myrtaceae & 1 & 1 & 0,05 & 0,02 & 0,09 & 0,16 & 0,07 \\
\hline Miconia sp. 1 & Melastomataceae & 1 & 1 & 0,05 & 0,01 & 0,09 & 0,16 & 0,07 \\
\hline Mollinedia glabra (Spreng.) Perkins & Monimiaceae & 1 & 1 & 0,05 & 0,01 & 0,09 & 0,16 & 0,07 \\
\hline Mollinedia sp. 1 & Monimiaceae & 1 & 1 & 0,05 & 0,02 & 0,09 & 0,16 & 0,08 \\
\hline Myrcia guianensis (Aubl.) DC. & Myrtaceae & 1 & 1 & 0,05 & 0,01 & 0,09 & 0,16 & 0,07 \\
\hline Pimenta pseudocaryophyllus (Gomes) Landrum & Myrtaceae & 1 & 1 & 0,05 & 0,01 & 0,09 & 0,16 & 0,07 \\
\hline Psychotria sp. 1 & Rubiaceae & 1 & 1 & 0,05 & 0,01 & 0,09 & 0,16 & 0,07 \\
\hline Randia armata (Sw.) DC. & Rubiaceae & 1 & 1 & 0,05 & 0,01 & 0,09 & 0,16 & 0,07 \\
\hline Casearia decandra Jacq. & Salicaceae & 1 & 1 & 0,05 & 0,01 & 0,09 & 0,15 & 0,06 \\
\hline Eugenia sp. 5 & Myrtaceae & 1 & 1 & 0,05 & 0,01 & 0,09 & 0,15 & 0,06 \\
\hline Eugenia sp. 6 & Myrtaceae & 1 & 1 & 0,05 & 0,01 & 0,09 & 0,15 & 0,06 \\
\hline Gomidesia sp. 1 & Myrtaceae & 1 & 1 & 0,05 & 0,01 & 0,09 & 0,15 & 0,06 \\
\hline Guatteria pohliana Schltdl. & Annonaceae & 1 & 1 & 0,05 & 0,01 & 0,09 & 0,15 & 0,06 \\
\hline Hieronyma alchorneoides Allemão & Phyllanthaceae & 1 & 1 & 0,05 & 0 & 0,09 & 0,15 & 0,06 \\
\hline Inga cf. capitata Desv. & Fabaceae & 1 & 1 & 0,05 & 0,01 & 0,09 & 0,15 & 0,07 \\
\hline Maytenus schumanniana Loes. & Celastraceae & 1 & 1 & 0,05 & 0,01 & 0,09 & 0,15 & 0,06 \\
\hline Mollinedia acutissima Perkins & Monimiaceae & 1 & 1 & 0,05 & 0,01 & 0,09 & 0,15 & 0,06 \\
\hline Mollinedia aff. glabra (Spreng.) Perkins & Monimiaceae & 1 & 1 & 0,05 & 0,01 & 0,09 & 0,15 & 0,06 \\
\hline Myrceugenia myrcioides (Cambess.) O.Berg & Myrtaceae & 1 & 1 & 0,05 & 0,01 & 0,09 & 0,15 & 0,06 \\
\hline Pera sp.1 & Euphorbiaceae & 1 & 1 & 0,05 & 0,01 & 0,09 & 0,15 & 0,06 \\
\hline Rapanea leuconeura (Mart.) Mez & Myrsinaceae & 1 & 1 & 0,05 & 0,01 & 0,09 & 0,15 & 0,06 \\
\hline Solanum pseudoquina A.St.-Hil. & Solanaceae & 1 & 1 & 0,05 & 0,01 & 0,09 & 0,15 & 0,06 \\
\hline Syzygium cf. jambos (L.) Alston & Myrtaceae & 1 & 1 & 0,05 & 0 & 0,09 & 0,15 & 0,06 \\
\hline Tetrorchidium parvulum Müll. Arg. & Euphorbiaceae & 1 & 1 & 0,05 & 0 & 0,09 & 0,15 & 0,06 \\
\hline Vernonanthura discolor (Spreng.) H.Rob. & Asteraceae & 1 & 1 & 0,05 & 0,01 & 0,09 & 0,15 & 0,06 \\
\hline Weinmannia paulliniifolia Pohl ex Ser. & Cunoniaceae & 1 & 1 & 0,05 & 0,01 & 0,09 & 0,15 & 0,06 \\
\hline
\end{tabular}

As espécies em cinza são consideradas exclusivas da área $\mathrm{K}$.

Species in gray color are considered unique to the area $\mathrm{K}$.

("Sobs Mao Tau") e mostrou que a parcela $\mathrm{K}$ teria mais espécies ( $\mathrm{K}=172,56$ e $\mathrm{N}=150$ espécies) (Figura 3). Porém, a estimativa máxima de espécies no ponto de rarefação da parcela N (IC 95\% - 158,54) se sobrepôs à estimativa mínima no mesmo ponto para a parcela K (IC 95\% - 157,12) e então o número de espécies de ambas as áreas se equivaleriam em 1.420 indivíduos.

Para heterogeneidade, o índice de Shannon-Weiner foi maior para a parcela $\mathrm{N}$ do que para a parcela $\mathrm{K}\left(\mathrm{H}^{\prime}=4,05\right.$ nats.ind $^{-1} \mathrm{e}$ $\mathrm{H}^{\prime}=3,72$ nats.ind ${ }^{-1}$ respectivamente), assim como a equabilidade $\left(\mathrm{J}^{\prime}=0,8\right.$ e J' $=0,7$ respectivamente) e o número efetivo de espécies obtido a partir dos valores do índice acima mencionado $(\mathrm{N}=57,39 \mathrm{e}$
$K=41,26$ ) (Tabela 3). Na comparação da composição de espécies, obtida pelo coeficiente qualitativo de Jaccard (Krebs 1999), as áreas mostraram baixa similaridade entre si $\left(\mathrm{C}_{\mathrm{J}}=0,3\right)$, ou seja, as amostras são semelhantes na densidade de espécies, mas distintas nas espécies que as compõem. Das 265 espécies encontradas, apenas 94 (35,5\%) são compartilhadas entre as áreas. Do restante, 69 (26\%) são exclusivas da parcela $\mathrm{N}$ e $102(38,5 \%)$ exclusivas da parcela $\mathrm{K}$ (Tabela 1 e 2).

A parcela $\mathrm{N}$ possui 149 espécies distribuídas em 40 famílias botânicas. As famílias com mais espécies foram Myrtaceae (27), Lauraceae (15), Fabaceae e Melastomataceae (oito espécies cada) 
Tabela 2. Fitossociologia da Parcela N (secundária). As espécies estão classificadas pelo valor decrescente de V.I. n.i. - número de indivíduos; n.a. - número de amostras em que a espécie ocorreu; De.R. -densidade relativa; Do.R. - dominância relativa; Fr.R.- frequência relativa; V.I. - valor de importância; V.C. valor de cobertura.

Table 2. Phytosociology of Plot N (secondary). Species are ranked by decreasing value of V.I. n.i. - number of individuals; n.a. - number of samples where the species occurred; De.R. - relative density; Do.R. - relative dominance; Fr.R. - relative frequency; V.I. - value of importance; V.C. - value coverage.

\begin{tabular}{|c|c|c|c|c|c|c|c|c|}
\hline Espécies & Família & n.i. & n.a. & De.R & Do.R & Fr.R & V.I. & V.C. \\
\hline Licania hoehnei Pilg. & Chrysobalanaceae & 42 & 30 & 2.92 & 8.61 & 3.00 & 14.54 & 11.54 \\
\hline Indeterminada sp. 1 & Indeterminada & 21 & 16 & 1.46 & 9.10 & 1.60 & 12.16 & 10.56 \\
\hline Mollinedia argyrogyna Perkins & Monimiaceae & 35 & 26 & 2.44 & 3.31 & 2.60 & 8.35 & 5.75 \\
\hline Chrysophyllum viride Mart. \& Eichler & Sapotaceae & 20 & 16 & 1.39 & 4.33 & 1.60 & 7.32 & 5.72 \\
\hline Myrcia spectabilis DC. & Myrtaceae & 37 & 25 & 2.58 & 1.04 & 2.50 & 6.12 & 3.62 \\
\hline Marlierea tomentosa Cambess. & Myrtaceae & 29 & 24 & 2.02 & 0.87 & 2.40 & 5.29 & 2.89 \\
\hline Calyptranthes strigipes O.Berg & Myrtaceae & 21 & 17 & 1.46 & 1.99 & 1.70 & 5.15 & 3.45 \\
\hline Miconia sp. 1 & Melastomataceae & 30 & 19 & 2.09 & 0.43 & 1.90 & 4.42 & 2.51 \\
\hline Cordia sellowiana Cham. & Boraginaceae & 19 & 19 & 1.32 & 1.05 & 1.90 & 4.28 & 2.37 \\
\hline Cyathea delgadii Sternb. & Cyatheaceae & 19 & 10 & 1.32 & 1.24 & 1.00 & 3.56 & 2.56 \\
\hline Matayba guianensis Aubl. & Sapindaceae & 23 & 16 & 1.60 & 0.29 & 1.60 & 3.50 & 1.89 \\
\hline Jacaranda montana Morawetz & Bignoniaceae & 9 & 9 & 0.63 & 1.80 & 0.90 & 3.33 & 2.43 \\
\hline Cryptocarya mandioccana Meisn. & Lauraceae & 15 & 13 & 1.04 & 0.94 & 1.30 & 3.28 & 1.98 \\
\hline Marlierea silvatica (O.Berg) Kiaersk. & Myrtaceae & 16 & 15 & 1.11 & 0.66 & 1.50 & 3.28 & 1.77 \\
\hline Ocotea dispersa (Nees \& Mart.) Mez & Lauraceae & 15 & 13 & 1.04 & 0.93 & 1.30 & 3.28 & 1.98 \\
\hline Cryptocarya saligna $\mathrm{Mez}$ & Lauraceae & 11 & 11 & 0.77 & 1.31 & 1.10 & 3.18 & 2.08 \\
\hline Hieronyma alchorneoides Allemão & Phyllanthaceae & 11 & 9 & 0.77 & 0.82 & 0.90 & 2.49 & 1.59 \\
\hline Hirtella hebeclada Moric. ex DC. & Chrysobalanaceae & 8 & 8 & 0.56 & 1.11 & 0.80 & 2.47 & 1.67 \\
\hline Cabralea canjerana subsp. canjerana (Vell.) Mart. & Meliaceae & 7 & 6 & 0.49 & 1.33 & 0.60 & 2.41 & 1.81 \\
\hline Guapira nitida (Mart. ex J.A.Schmidt) Lundell & Nyctaginaceae & 14 & 13 & 0.97 & 0.13 & 1.30 & 2.41 & 1.11 \\
\hline Guatteria australis A.St.-Hil. & Annonaceae & 12 & 12 & 0.84 & 0.37 & 1.20 & 2.41 & 1.21 \\
\hline Campomanesia guaviroba (DC.) Kiaersk. & Myrtaceae & 7 & 6 & 0.49 & 1.28 & 0.60 & 2.37 & 1.76 \\
\hline Cyathea phalerata Mart. & Cyatheaceae & 13 & 6 & 0.91 & 0.79 & 0.60 & 2.29 & 1.69 \\
\hline Mollinedia elegans Tul. & Monimiaceae & 13 & 12 & 0.91 & 0.15 & 1.20 & 2.26 & 1.06 \\
\hline Indeterminada sp. 2 & Indeterminada & 10 & 8 & 0.70 & 0.73 & 0.80 & 2.23 & 1.42 \\
\hline Myrciaria floribunda (H.West ex Willd.) O.Berg & Myrtaceae & 12 & 11 & 0.84 & 0.24 & 1.10 & 2.18 & 1.08 \\
\hline Alsophila sternbergii (Sternb.) D.S.Conant & Cyatheaceae & 10 & 6 & 0.70 & 0.82 & 0.60 & 2.12 & 1.52 \\
\hline Casearia cf. obliqua Spreng. & Salicaceae & 2 & 2 & 0.14 & 1.70 & 0.20 & 2.04 & 1.84 \\
\hline Guapira areolata (Heimerl) Lundell & Nyctaginaceae & 9 & 7 & 0.63 & 0.24 & 0.70 & 1.57 & 0.87 \\
\hline Eugenia prasina O.Berg & Myrtaceae & 9 & 8 & 0.63 & 0.13 & 0.80 & 1.56 & 0.76 \\
\hline Casearia paranaensis Sleumer & Salicaceae & 6 & 4 & 0.42 & 0.72 & 0.40 & 1.54 & 1.14 \\
\hline Myrcia splendens (Sw.) DC. & Myrtaceae & 10 & 7 & 0.70 & 0.13 & 0.70 & 1.53 & 0.82 \\
\hline Vernonanthura puberula (Less.) H. Rob. & Asteraceae & 4 & 4 & 0.28 & 0.83 & 0.40 & 1.51 & 1.11 \\
\hline Myrtaceae sp. 2 & Myrtaceae & 5 & 5 & 0.35 & 0.63 & 0.50 & 1.48 & 0.98 \\
\hline Schefflera calva (Cham.) Frodin \& Fiaschi & Araliaceae & 8 & 8 & 0.56 & 0.09 & 0.80 & 1.44 & 0.64 \\
\hline Schefflera angustissima (Marchal) Frodin & Araliaceae & 7 & 6 & 0.49 & 0.30 & 0.60 & 1.38 & 0.78 \\
\hline Inga sessilis (Vell.) Mart. & Fabaceae & 4 & 4 & 0.28 & 0.66 & 0.40 & 1.34 & 0.94 \\
\hline Eugenia cuprea (O.Berg) Nied. & Myrtaceae & 7 & 5 & 0.49 & 0.34 & 0.50 & 1.33 & 0.83 \\
\hline Calyptranthes sp. 2 & Myrtaceae & 6 & 6 & 0.42 & 0.29 & 0.60 & 1.31 & 0.71 \\
\hline Eugenia batingabranca Sobral & Myrtaceae & 7 & 6 & 0.49 & 0.22 & 0.60 & 1.31 & 0.71 \\
\hline Pouteria gardneri (Mart. \& Miq.) Baehni & Sapotaceae & 3 & 3 & 0.21 & 0.76 & 0.30 & 1.27 & 0.97 \\
\hline Sloanea monosperma Vell. & Elaeocarpaceae & 6 & 6 & 0.42 & 0.21 & 0.60 & 1.23 & 0.63 \\
\hline Stephanopodium estrellense Baill. & Dichapetalaceae & 6 & 6 & 0.42 & 0.21 & 0.60 & 1.23 & 0.63 \\
\hline Eugenia oblongata O.Berg & Myrtaceae & 6 & 6 & 0.42 & 0.11 & 0.60 & 1.13 & 0.52 \\
\hline Mollinedia ovata Ruiz \& Pav. & Monimiaceae & 6 & 6 & 0.42 & 0.11 & 0.60 & 1.13 & 0.53 \\
\hline Daphnopsis schwackeana Taub. & Thymelaeaceae & 6 & 6 & 0.42 & 0.10 & 0.60 & 1.12 & 0.52 \\
\hline Ficus sp. 1 & Moraceae & 2 & 2 & 0.14 & 0.73 & 0.20 & 1.07 & 0.87 \\
\hline Vernonanthura aff. puberula (Less.) H. Rob. & Asteraceae & 5 & 5 & 0.35 & 0.17 & 0.50 & 1.02 & 0.52 \\
\hline
\end{tabular}

As espécies em cinza são consideradas exclusivas da área $\mathrm{N}$.

Species in gray color are considered unique to the area $\mathrm{N}$. 
Tabela 2. Continuação...

\begin{tabular}{|c|c|c|c|c|c|c|c|c|}
\hline Espécies & Família & n.i. & n.a. & De.R & Do.R & Fr.R & V.I. & V.C. \\
\hline Psychotria longipes Müll.Arg. & Rubiaceae & 5 & 5 & 0.35 & 0.16 & 0.50 & 1.01 & 0.51 \\
\hline Myrtaceae sp. 1 & Myrtaceae & 2 & 2 & 0.14 & 0.66 & 0.20 & 1.00 & 0.80 \\
\hline Eugenia cerasiflora Miq. & Myrtaceae & 3 & 3 & 0.21 & 0.08 & 0.30 & 0.59 & 0.29 \\
\hline Miconia cabucu Hoehne & Melastomataceae & 3 & 3 & 0.21 & 0.08 & 0.30 & 0.59 & 0.29 \\
\hline Ocotea glaziovii $\mathrm{Mez}$ & Lauraceae & 3 & 3 & 0.21 & 0.05 & 0.30 & 0.56 & 0.26 \\
\hline Posoqueria latifolia (Rudge) Schult. & Rubiaceae & 3 & 3 & 0.21 & 0.05 & 0.30 & 0.56 & 0.26 \\
\hline Eugenia tinguyensis Cambess. & Myrtaceae & 3 & 3 & 0.21 & 0.04 & 0.30 & 0.55 & 0.25 \\
\hline Psychotria vellosiana Benth. & Rubiaceae & 3 & 3 & 0.21 & 0.04 & 0.30 & 0.55 & 0.25 \\
\hline Myrcia guianensis (Aubl.) DC. & Myrtaceae & 3 & 3 & 0.21 & 0.02 & 0.30 & 0.53 & 0.23 \\
\hline Piper cernuum Vell. & Piperaceae & 3 & 3 & 0.21 & 0.02 & 0.30 & 0.53 & 0.23 \\
\hline Rutaceae sp. 1 & Rutaceae & 2 & 2 & 0.14 & 0.17 & 0.20 & 0.51 & 0.31 \\
\hline Inga marginata Willd. & Fabaceae & 2 & 2 & 0.14 & 0.16 & 0.20 & 0.50 & 0.30 \\
\hline Cecropia glaziovii Snethl. & Urticaceae & 2 & 2 & 0.14 & 0.15 & 0.20 & 0.49 & 0.29 \\
\hline Senna macranthera (DC. ex Collad.) H.S.Irwin \& Barneby & Fabaceae & 2 & 2 & 0.14 & 0.14 & 0.20 & 0.48 & 0.28 \\
\hline Chrysophyllum inornatum Mart. & Sapotaceae & 2 & 1 & 0.14 & 0.21 & 0.10 & 0.45 & 0.35 \\
\hline Myrtaceae sp. 9 & Myrtaceae & 1 & 1 & 0.07 & 0.28 & 0.10 & 0.45 & 0.35 \\
\hline Swartzia sp. 1 & Fabaceae & 1 & 1 & 0.07 & 0.27 & 0.10 & 0.44 & 0.34 \\
\hline Cupania oblongifolia Mart. & Sapindaceae & 2 & 2 & 0.14 & 0.09 & 0.20 & 0.43 & 0.23 \\
\hline Aureliana fasciculata var. longifolia (Sendtn.) Hunz. \& Barboza & Solanaceae & 2 & 2 & 0.14 & 0.08 & 0.20 & 0.42 & 0.22 \\
\hline Aniba viridis $\mathrm{Mez}$ & Lauraceae & 2 & 2 & 0.14 & 0.04 & 0.20 & 0.38 & 0.18 \\
\hline Chomelia pedunculosa Benth. & Rubiaceae & 2 & 2 & 0.14 & 0.03 & 0.20 & 0.37 & 0.17 \\
\hline Maytenus littoralis Carv.-Okano & Celastraceae & 2 & 2 & 0.14 & 0.03 & 0.20 & 0.37 & 0.17 \\
\hline Solanum cf. pseudoquina A.St.-Hil. & Solanaceae & 2 & 2 & 0.14 & 0.03 & 0.20 & 0.37 & 0.17 \\
\hline Trichilia silvatica C.DC. & Meliaceae & 1 & 1 & 0.07 & 0.20 & 0.10 & 0.37 & 0.27 \\
\hline Vernonanthura discolor (Spreng.) H.Rob. & Asteraceae & 2 & 1 & 0.14 & 0.13 & 0.10 & 0.37 & 0.27 \\
\hline Cryptocarya moschata Nees \& Mart. & Lauraceae & 2 & 2 & 0.14 & 0.02 & 0.20 & 0.36 & 0.16 \\
\hline Cupania vernalis Cambess. & Sapindaceae & 2 & 2 & 0.14 & 0.02 & 0.20 & 0.36 & 0.15 \\
\hline Vantanea compacta (Schnizl.) Cuatrec. & Humiriaceae & 2 & 2 & 0.14 & 0.02 & 0.20 & 0.36 & 0.16 \\
\hline Eugenia sp. 4 & Myrtaceae & 2 & 2 & 0.14 & 0.01 & 0.20 & 0.35 & 0.15 \\
\hline Solanum swartzianum Roem. \& Schult. & Solanaceae & 3 & 1 & 0.21 & 0.04 & 0.10 & 0.35 & 0.25 \\
\hline Solanum vellozianum Dunal & Solanaceae & 2 & 2 & 0.14 & 0.01 & 0.20 & 0.35 & 0.15 \\
\hline Mollinedia sp. 1 & Monimiaceae & 1 & 1 & 0.07 & 0.17 & 0.10 & 0.34 & 0.24 \\
\hline Solanum aff. rufescens Sendtn. & Solanaceae & 3 & 1 & 0.21 & 0.02 & 0.10 & 0.33 & 0.23 \\
\hline Coussapoa sp. 2 & Urticaceae & 1 & 1 & 0.07 & 0.10 & 0.10 & 0.27 & 0.17 \\
\hline Solanum sp. 1 & Solanaceae & 2 & 1 & 0.14 & 0.01 & 0.10 & 0.25 & 0.15 \\
\hline Psychotria brachypoda (Müll.Arg.) Britton & Rubiaceae & 1 & 1 & 0.07 & 0.07 & 0.10 & 0.24 & 0.14 \\
\hline Quiina magallano-gomesii Schwacke & Quiinaceae & 1 & 1 & 0.07 & 0.07 & 0.10 & 0.24 & 0.14 \\
\hline Maytenus sp. 6 & Celastraceae & 1 & 1 & 0.07 & 0.06 & 0.10 & 0.23 & 0.13 \\
\hline Tibouchina sp. 1 & Melastomataceae & 1 & 1 & 0.07 & 0.05 & 0.10 & 0.22 & 0.12 \\
\hline Melastomataceae sp. 1 & Melastomataceae & 1 & 1 & 0.07 & 0.04 & 0.10 & 0.21 & 0.11 \\
\hline Ocotea silvestris Vattimo-Gil & Lauraceae & 1 & 1 & 0.07 & 0.04 & 0.10 & 0.21 & 0.11 \\
\hline Coussapoa sp. 1 & Urticaceae & 1 & 1 & 0.07 & 0.03 & 0.10 & 0.20 & 0.10 \\
\hline Cinnamomum triplinerve (Ruiz \& Pav.) Kosterm. & Lauraceae & 1 & 1 & 0.07 & 0.02 & 0.10 & 0.19 & 0.09 \\
\hline Ecclinusa ramiflora Mart. & Sapotaceae & 1 & 1 & 0.07 & 0.02 & 0.10 & 0.19 & 0.09 \\
\hline Hedyosmum brasiliense Miq. & Chloranthaceae & 1 & 1 & 0.07 & 0.02 & 0.10 & 0.19 & 0.09 \\
\hline Miconia sp. 2 & Melastomataceae & 1 & 1 & 0.07 & 0.02 & 0.10 & 0.19 & 0.09 \\
\hline Agonandra excelsa Griseb. & Opiliaceae & 1 & 1 & 0.07 & 0.01 & 0.10 & 0.18 & 0.08 \\
\hline Cedrela odorata $\mathrm{L}$. & Meliaceae & 1 & 1 & 0.07 & 0.01 & 0.10 & 0.18 & 0.08 \\
\hline Cryptocarya sp. 1 & Lauraceae & 1 & 1 & 0.07 & 0.01 & 0.10 & 0.18 & 0.08 \\
\hline Eugenia burkartiana (D.Legrand) D.Legrand & Myrtaceae & 1 & 1 & 0.07 & 0.01 & 0.10 & 0.18 & 0.08 \\
\hline Euplassa hoehnei Sleumer & Proteaceae & 1 & 1 & 0.07 & 0.01 & 0.10 & 0.18 & 0.08 \\
\hline
\end{tabular}

As espécies em cinza são consideradas exclusivas da área $\mathrm{N}$.

Species in gray color are considered unique to the area $\mathrm{N}$. 
Tabela 2. Continuação...

\begin{tabular}{|c|c|c|c|c|c|c|c|c|}
\hline Espécies & Família & n.i. & n.a. & De.R & Do.R & Fr.R & V.I. & V.C. \\
\hline Meliosma sellowii Urb. & Sabiaceae & 1 & 1 & 0.07 & 0.01 & 0.10 & 0.18 & 0.08 \\
\hline Miconia aff. valtheri Naudin & Melastomataceae & 1 & 1 & 0.07 & 0.01 & 0.10 & 0.18 & 0.08 \\
\hline Nectandra psammophila Nees & Lauraceae & 1 & 1 & 0.07 & 0.01 & 0.10 & 0.18 & 0.08 \\
\hline Ocotea sp. 1 & Lauraceae & 1 & 1 & 0.07 & 0.01 & 0.10 & 0.18 & 0.08 \\
\hline Rubiaceae sp. 1 & Rubiaceae & 1 & 1 & 0.07 & 0.01 & 0.10 & 0.18 & 0.08 \\
\hline Solanum cinnamomeum Sendtn. & Solanaceae & 1 & 1 & 0.07 & 0.01 & 0.10 & 0.18 & 0.08 \\
\hline Mollinedia schottiana (Spreng.) Perkins & Monimiaceae & 25 & 18 & 1.74 & 0.44 & 1.80 & 3.98 & 2.18 \\
\hline Mollinedia glabra (Spreng.) Perkins & Monimiaceae & 13 & 8 & 0.91 & 0.27 & 0.80 & 1.98 & 1.18 \\
\hline Eugenia sp. 1 & Myrtaceae & 18 & 14 & 1.25 & 0.28 & 1.40 & 2.93 & 1.53 \\
\hline Bathysa australis (A.St.-Hil.) K.Schum. & Rubiaceae & 26 & 17 & 1.81 & 1.41 & 1.70 & 4.92 & 3.22 \\
\hline Licaria armeniaca (Nees) Kosterm. & Lauraceae & 18 & 16 & 1.25 & 1.06 & 1.60 & 3.91 & 2.31 \\
\hline Micropholis gardneriana (A.DC.) Pierre & Sapotaceae & 5 & 4 & 0.35 & 1.14 & 0.40 & 1.89 & 1.49 \\
\hline Alchornea glandulosa Poepp. \& Endl. & Euphorbiaceae & 24 & 12 & 1.67 & 0.98 & 1.20 & 3.85 & 2.65 \\
\hline Pouteria caimito (Ruiz \& Pav.) Radlk. & Sapotaceae & 17 & 16 & 1.18 & 1.06 & 1.60 & 3.85 & 2.25 \\
\hline Croton macrobothrys Baill. & Euphorbiaceae & 6 & 6 & 0.42 & 0.83 & 0.60 & 1.84 & 1.24 \\
\hline Calyptranthes lucida Mart. ex DC. & Myrtaceae & 40 & 28 & 2.79 & 2.19 & 2.80 & 7.78 & 4.98 \\
\hline Alsophila setosa Kaulf. & Cyatheaceae & 17 & 9 & 1.18 & 1.68 & 0.90 & 3.76 & 2.86 \\
\hline Inga lanceifolia Benth. & Fabaceae & 21 & 19 & 1.46 & 2.39 & 1.90 & 5.75 & 3.85 \\
\hline Mollinedia engleriana Perkins & Monimiaceae & 21 & 18 & 1.46 & 1.44 & 1.80 & 4.71 & 2.90 \\
\hline Myrceugenia myrcioides (Cambess.) O.Berg & Myrtaceae & 10 & 8 & 0.70 & 0.15 & 0.80 & 1.65 & 0.85 \\
\hline Guarea macrophylla subsp. tuberculata (Vell.) T.D.Penn. & Meliaceae & 20 & 15 & 1.39 & 0.74 & 1.50 & 3.64 & 2.14 \\
\hline Ocotea catharinensis $\mathrm{Mez}$ & Lauraceae & 37 & 25 & 2.58 & 3.55 & 2.50 & 8.63 & 6.13 \\
\hline Alchornea triplinervia (Spreng.) Müll.Arg. & Euphorbiaceae & 22 & 16 & 1.53 & 5.49 & 1.60 & 8.62 & 7.02 \\
\hline Tibouchina pulchra Cogn. & Melastomataceae & 8 & 7 & 0.56 & 0.37 & 0.70 & 1.62 & 0.92 \\
\hline Piptocarpha macropoda (DC.) Baker & Asteraceae & 5 & 5 & 0.35 & 0.75 & 0.50 & 1.60 & 1.10 \\
\hline Rapanea hermogenesii Jung-Mend. \& Bernacci & Myrsinaceae & 8 & 8 & 0.56 & 0.24 & 0.80 & 1.60 & 0.80 \\
\hline Annona dolabripetala Raddi & Annonaceae & 4 & 3 & 0.28 & 0.40 & 0.30 & 0.98 & 0.68 \\
\hline Vernonanthura petiolaris (DC.) H.Rob. & Asteraceae & 3 & 2 & 0.21 & 0.53 & 0.20 & 0.94 & 0.74 \\
\hline Maytenus robusta Reissek & Celastraceae & 5 & 4 & 0.35 & 0.17 & 0.40 & 0.91 & 0.51 \\
\hline Guarea kunthiana A.Juss. & Meliaceae & 6 & 4 & 0.42 & 0.08 & 0.40 & 0.90 & 0.50 \\
\hline Lauraceae sp. 2 & Lauraceae & 3 & 3 & 0.21 & 0.40 & 0.30 & 0.90 & 0.60 \\
\hline Myrtaceae sp. 3 & Myrtaceae & 2 & 2 & 0.14 & 0.53 & 0.20 & 0.87 & 0.67 \\
\hline Cyathea atrovirens (Langsd. \& Fisch.) Domin & Cyatheaceae & 5 & 4 & 0.35 & 0.09 & 0.40 & 0.84 & 0.44 \\
\hline Guapira opposita (Vell.) Reitz & Nyctaginaceae & 3 & 3 & 0.21 & 0.29 & 0.30 & 0.80 & 0.50 \\
\hline Eugenia sp. 3 & Myrtaceae & 3 & 3 & 0.21 & 0.26 & 0.30 & 0.76 & 0.46 \\
\hline Pterocarpus rohrii Vahl & Fabaceae & 4 & 4 & 0.28 & 0.07 & 0.40 & 0.75 & 0.35 \\
\hline Calyptranthes rufa O.Berg & Myrtaceae & 4 & 4 & 0.28 & 0.06 & 0.40 & 0.74 & 0.34 \\
\hline Cyathea dichromatolepis (Fée) Domin & Cyatheaceae & 4 & 4 & 0.28 & 0.07 & 0.40 & 0.74 & 0.34 \\
\hline Mouriri chamissoana Cogn. & Memecylaceae & 3 & 3 & 0.21 & 0.24 & 0.30 & 0.74 & 0.44 \\
\hline Coussapoa microcarpa (Schott) Rizzini & Urticaceae & 3 & 3 & 0.21 & 0.22 & 0.30 & 0.73 & 0.43 \\
\hline Heisteria silvianii Schwacke & Olacaceae & 4 & 4 & 0.28 & 0.05 & 0.40 & 0.73 & 0.33 \\
\hline Rapanea gardneriana (A. DC.) Mez & Myrsinaceae & 4 & 3 & 0.28 & 0.14 & 0.30 & 0.72 & 0.42 \\
\hline Citronella megaphylla (Miers) R.A.Howard & Cardiopteridaceae & 3 & 3 & 0.21 & 0.20 & 0.30 & 0.71 & 0.41 \\
\hline Cupania furfuracea Radlk. & Sapindaceae & 2 & 2 & 0.14 & 0.36 & 0.20 & 0.70 & 0.50 \\
\hline Machaerium brasiliense Vogel & Fabaceae & 1 & 1 & 0.07 & 0.53 & 0.10 & 0.70 & 0.60 \\
\hline Pimenta pseudocaryophyllus (Gomes) Landrum & Myrtaceae & 2 & 2 & 0.14 & 0.32 & 0.20 & 0.66 & 0.46 \\
\hline Machaerium nyctitans (Vell.) Benth. & Fabaceae & 1 & 1 & 0.07 & 0.46 & 0.10 & 0.63 & 0.53 \\
\hline Casearia cf. sylvestris Sw. & Salicaceae & 3 & 3 & 0.21 & 0.10 & 0.30 & 0.61 & 0.31 \\
\hline Ocotea daphnifolia (Meisn.) Mez & Lauraceae & 2 & 2 & 0.14 & 0.26 & 0.20 & 0.60 & 0.40 \\
\hline Euterpe edulis Mart. & Arecaceae & 298 & 82 & 20.75 & 9.19 & 8.21 & 38.15 & 29.94 \\
\hline
\end{tabular}

As espécies em cinza são consideradas exclusivas da área $\mathrm{N}$.

Species in gray color are considered unique to the area $\mathrm{N}$. 
e Monimiaceae (sete). Exceto Fabaceae e Melastomataceae, as demais famílias foram também as de maior densidade, acrescentando Arecaceae ao ranking (Tabela 2). O gênero Miconia (Melastomataceae) teve maior abundância nesta parcela do que na área K (35 e 23, respectivamente). Myrtaceae, Lauraceae e Solanaceae somaram mais espécies exclusivas na área (oito para a primeira e seis para cada uma das demais). Embora Asteraceae tenha apenas duas espécies exclusivas (12 indivíduos), a soma das dominâncias relativas (Do. $\mathrm{R}=1,53)$ foi mais que a metade da soma para Lauraceae na mesma área, que contribui com seis espécies e 41 indivíduos (Do.R = 2,34). A soma das dominâncias das espécies de Fabaceae foi alta também (15 ind., Do.R =2,29). As espécies exclusivas mais abundantes foram Alchornea triplinervia (Spreng.) Müll. Arg. (22) e Licaria armeniaca (Ness) Kosteamans (Tabela 2).

Para a parcela K, as 189 espécies encontradas distribuíram-se em 43 famílias botânicas. A família de maior densidade foi Arecaceae (562 indivíduos), seguida de Myrtaceae (295), Lauraceae (142) e Sapotaceae (103) (Tabela 1). Porém, a primeira família apresentou apenas uma espécie, E.edulis. Já Myrtaceae e Lauraceae apareceram

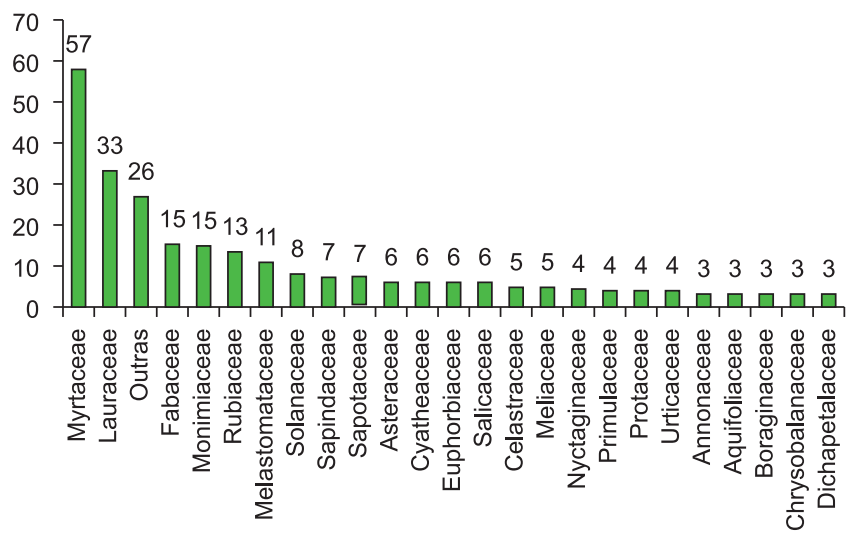

Figura 1. Distribuição da densidade de espécies por família (soma dos 2 ha). O item "outras" inclui as famílias com uma ou duas espécies: Arecaceae, Bignoniaceae, Caricaceae, Chloranthaceae, Humiriaceae, Malpighiaceae, Memecylaceae, Moraceae, Ochnaceae, Olacaceae, Oleaceae, Opiliaceae, Phyllanthaceae, Phytolacaceae, Piperaceae, Quiinaceae, Rosaceae, Sabiaceae, Thymelaeaceae, Winteraceae, Araliaceae, Cardiopteridaceae, Clusiaceae, Cunoniaceae, Elaeocarpaceae, Rutaceae, Symplocaceae.

Figure 1. Density distribution of species per family (sum of 2 ha). The "other" includes families with one or two species: Arecaceae, Bignoniaceae, Caricaceae, Chloranthaceae, Humiriaceae, Malpighiaceae, Memecylaceae, Moraceae, Ochnaceae, Olacaceae, Oleaceae, Opiliaceae, Phyllanthaceae, Phytolacaceae, Piperaceae, Quiinaceae, Rosaceae, Sabiaceae, Thymelaeaceae, Winteraceae, Araliaceae, Cardiopteridaceae, Clusiaceae, Cunoniaceae, Elaeocarpaceae, Rutaceae, Symplocaceae.

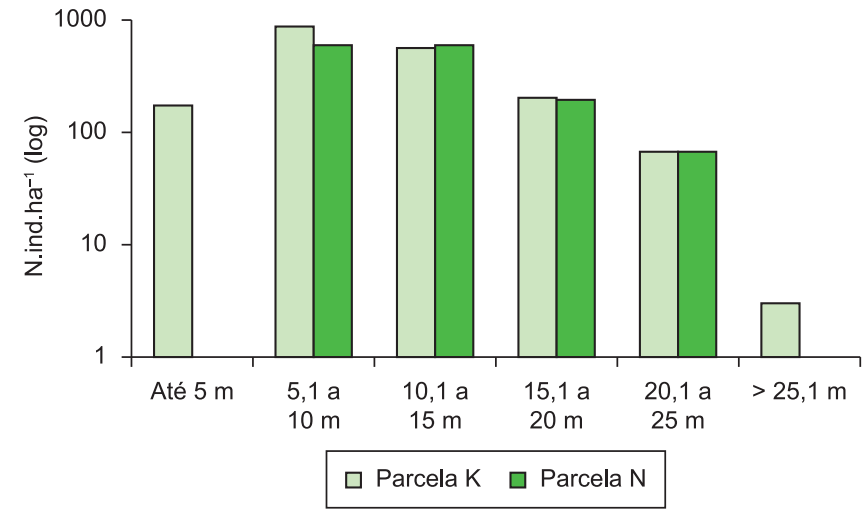

Figura 2. Distribuição das frequências das classes de tamanho (altura em metros). O eixo y representa o número de indivíduos em escala logarítmica. Em verde escuro parcela $\mathrm{N}$ (secundária); parcela $\mathrm{K}$ em verde claro (antiga).

Figure 2. Distribution of frequencies of size classes (height in meters). The $\mathrm{y}$-axis represents the number of individuals in logarithmic scale. Dark green portion $\mathrm{N}$ (secondary); plot $\mathrm{K}$ in light green (old).

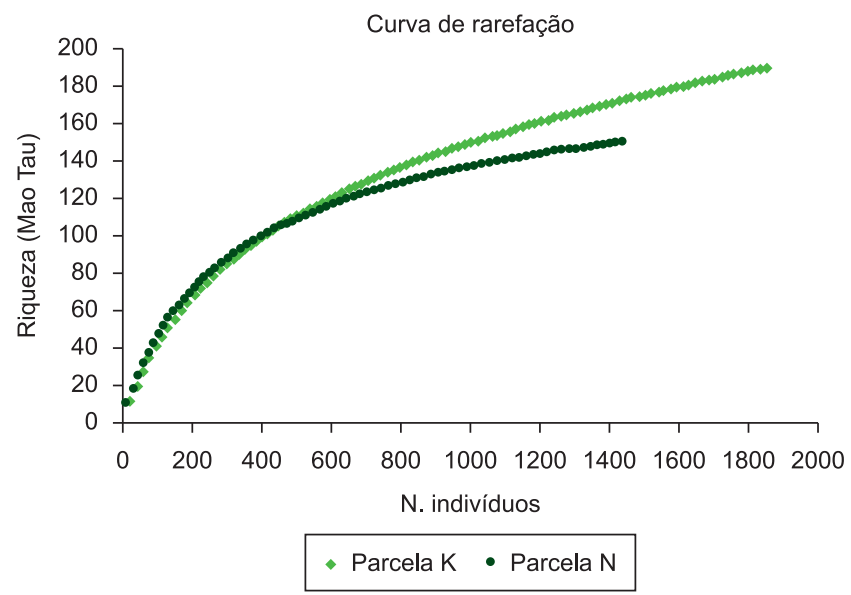

Figura 3. Curva de rarefação para as parcelas estudadas. O eixo y representa o número de indivíduos estimado com base na fórmula analítica "Mao Tau" (Colwell et al. 2004, Mao \& Colwell 2005). Em verde escuro parcela N (secundária); parcela $\mathrm{K}$ em verde claro (antiga).

Figure 3. Rarefaction curves for the plots studied. The y-axis represents the number of individuals estimated based on analytical formula 'Mao Tau' (Colwell et al. 2004, Mao \& Colwell 2005). Dark green portion N (secondary); portion $\mathrm{K}$ in light green (old)

Tabela 3. Comparação dos parâmetros estruturais (incluso densidade de espécies) analisados nas áreas de estudo (Parcelas K \& N).

Table 3. Comparison of structural parameters (including density of species) analyzed in the study areas (Plots K \& N).

\begin{tabular}{lccc}
\hline \multicolumn{1}{c}{ Parâmetro } & Parcela K & Parcela N & Geral $(\mathbf{K}+\mathbf{N})$ \\
\hline Área basal total $\left(\mathrm{m}^{2} \cdot\right.$ ha $\left.^{-1}\right)$ & 40,20 & 37,47 & 77,67 \\
Densidade de árvores vivas & 1851 & 1436 & 3287 \\
Altura média (m) & 9,1 & 11,7 & 10,2 \\
Diâmetro médio (cm $\left.{ }^{2}\right)$ & 12,74 & 14,29 & 13,42 \\
Shannon-Weiner (H') (nats.ind $\left.{ }^{-1}\right)$ & 3,72 & 4,06 & 4,12 \\
Equabilidade (J') & 0,7 & 0,8 & 0,73 \\
$\mathrm{~N}^{\circ}$ efetivo espécies (Jost 2006) & 41,42 & 58,03 & - \\
Densidade de espécies (ponto de rarefação) & 172,56 & 150 & - \\
$\mathrm{N}^{\circ}$ espécies com 1 ou 2 ind. & 92 & 56 & - \\
\hline
\end{tabular}


como as de maior densidade de espécies (48 e 26 respectivamente), seguidas de Monimiaceae (13), Rubiaceae (nove) e Fabaceae (oito). As famílias de maior contribuição para espécies exclusivas foram Myrtaceae (22), Lauraceae (15) e Monimiaceae (seis). Embora Myrtaceae tenha apresentado quase três vezes mais indivíduos de espécies exclusivas $(140$, Do. $R=5,63)$ do que a segunda colocada Lauraceae (50), esta teve a maior soma das dominâncias relativas de espécies exclusivas (Do.R = 5,99) (Tabela 1). Este resultado mostra que as espécies exclusivas pertencentes à última família têm área basal muito superior quando comparadas à Myrtaceae. No outro extremo estão duas famílias com baixa contribuição de espécies exclusivas e dominância relativa, mas que não estão presentes na parcela N, Symplocaceae (duas espécies, oito indivíduos, Do.R = 0,11 ) e Winteraceae (uma espécie, cinco indivíduos, Do.R =0,71). As espécies exclusivas de maior abundância foram Mollinedia salicifolia Perkins (23) e Couepia venosa Prance (19). A maior parte das espécies da parcela K (90) possuem entre um e dois indivíduos apenas.

\section{Discussão}

Do ponto de vista florístico, a área de MA estudada é tipicamente Floresta Montana, uma vez que as altas densidades de indivíduos nas famílias Arecaceae, Myrtaceae, Lauraceae, Monimiaceae e Cyatheaceae, são comuns nesse fitofisionomia (Robim et al. 1990, Mantovani 1993, Fontes 1997, Oliveira-Filho \& Fontes 2000, Tabarelli \& Mantovani, 2000, Guilherme et al. 2004, Dias 2005, Bertoncello 2009). Destaque para Monimiaceae que tem importância crescente no sudeste do país (Peixoto 1987), com particular tendência ao aumento a partir do nordeste do estado de São Paulo (Padgurschi 2010). Cyatheaceae foi a única família dentre os fetos arborescentes e ocorre, principalmente, em áreas de solo ácido situação característica das parcelas estudadas (Tonhasca Júnior 2005).

Com exceção de Arecaceae, as demais famílias supracitadas apresentaram alto número de espécies. Fabaceae e Rubiaceae também apresentaram muitas espécies, porém, com baixa densidade de indivíduos comparada às demais. A presença de taquaras (M. neesii) registrada para a região também é um importante componente de Florestas Montanas Atlânticas (Oliveira-Filho et al. 1994, Padgurschi 2010). Myrceugenia myrcioides (Cambess.) O.Berg (Myrtaceae), E.edulis, Ocotea catharinensis Mez (Lauraceae), Mollinedia argyrogyna e Mollinedia schottiana (Spreng.) Perkins (Monimiaceae) são espécies típicas de MA (Peixoto 1987, Falkenberg \& Voltolini 1993, Oliveira-Filho \& Fontes 2000, Barbosa 2009). Os resultados dos índices de Shannon-Weiner e Equabilidade de Pielou mostraram que, em conjunto, as áreas são bastante diversas, mas a distribuição dos indivíduos pelas espécies não é equitativa. A parcela $\mathrm{K}$ apresentou quase o dobro de espécies com um ou dois indivíduos apenas quando comparada à $\mathrm{N}$, que obteve equabilidade maior. Isto mostra que os indivíduos desta parcela estão distribuídos de maneira mais homogenia dentre as espécies. Esta situação é considerada típica de florestas secundárias, nas quais os táxons são melhores distribuídos (Brown \& Lugo 1990, Richard 1996).

A observação dos resultados para índice de Shannon-Weiner, número efetivo de espécies e estimativa baseada na rarefação, mostra que a parcela $\mathrm{N}$, considerada secundária, apresenta densidade de espécies equivalente à parcela $\mathrm{K}$. Relatos dos moradores indicam que a floresta $\mathrm{N}$ teria sofrido uma alteração moderada (L.S. Pereira, dados não publicados) e há mais de 25 anos está em processo de regeneração natural. Quando isto ocorre e tais áreas estão próximas a florestas mais antigas, o número de espécies lenhosas aumenta rapidamente durante os primeiros anos de sucessão e não leva mais do que algumas décadas para alcançar valores comparáveis aos de uma floresta madura (Finegan 1996, Guariguata \& Ostertag 2001). Os demais parâmetros estruturais também seguem esta tendência.

Para área basal e altura média também não houve diferenças significativas, isto porque o processo de maturidade da vegetação envolve mudanças estruturais rápidas (Brown \& Lugo 1990). Por outro lado, a estratificação vertical torna-se mais evidente somente com o aumento da idade das florestas, aumentando também a complexidade estrutural da comunidade (Oliveira Filho \& Fontes 2000, Guilherme et al. 2004, Liebsch et al. 2007). A parcela K apresentou dois estratos verticais a mais do que a parcela N (Figura 2). $\mathrm{O}$ padrão reflete a maturidade da parcela $\mathrm{N}$, onde as árvores se desenvolveram rapidamente no momento inicial de regeneração quando a copa ainda não estava fechada (Guariguata \& Ostertag 2001). Cyatheaceae dominou a classe de altura até $5 \mathrm{~m}$ (apenas 13 indivíduos ficaram na classe seguinte) na parcela $\mathrm{K}$, enquanto na parcela $\mathrm{N}$ nenhum indivíduo foi registrado. O domínio da família nesta classe foi registrado em outros trabalhos (Gentry 1995, Lacerda 2001).

De maneira geral, a recuperação da estrutura florestal em áreas secundárias ocorre mais rápido do que a composição (Guariguata \& Ostertag 2001, Alves \& Metzger 2006). O grau de similaridade florística entre as parcelas foi muito baixo. Esta diferença na composição, mas semelhança no número de espécies, pode ser explicada uma vez que ambos os parâmetros variam independentemente, ou seja, duas áreas podem ter táxons diferentes, mas número de espécies semelhantes (Finegan 1996).

As famílias mais abundantes foram praticamente as mesmas, exceto para a presença de Sapotaceae na parcela K. É à esta família que pertence Chrysophyllum viride Mart. \& Eichler, espécie explorada comercialmente na parcela N. A espécie está presente nesta área, porém em abundância e com dominância relativa muito menor. As famílias Fabaceae e Asteraceae contribuíram para ocorrência de espécies exclusivas na área $\mathrm{N}$ e a abundância dos gêneros Inga e Miconia nesta parcela são um indicativo de que a floresta é secundária (Richard 1996). Os gêneros ocorrem também na parcela K, mas em menor densidade e que pode ser comum nas clareiras de florestas antigas (Richard 1996). A espécie A. triplinervia, que não ocorreu na parcela $\mathrm{K}$, foi a que obteve maior densidade dentre as exclusivas na floresta $\mathrm{N}$, sendo também espécie de crescimento rápido.

O número de espécies de Monimiaceae foi alto na amostra N, mas a K apresentou quase o dobro de espécies. M. salicifolia, exclusiva e dominante na parcela $\mathrm{K}$, pertence às Monimiáceas e é sensível às perturbações naturais ou antrópicas na comunidade (Peixoto 1987). Outra família sensível a tais perturbações (J. Aranha com. pes.) é Symplocaceae. Mesmo com baixa dominância relativa na floresta K, a família contou com oito indivíduos, oposto à parcela $\mathrm{N}$, onde não houve registros para a mesma.

As áreas de estudo não diferem quanto à estrutura (incluindo aqui densidade de espécies). Este resultado era esperado, já que a área considerada secundária está em processo de regeneração natural há mais de 25 anos e que a intervenção foi moderada. Além disso, a proximidade entre as áreas secundária e antiga favorece a velocidade do poder regenerativo da floresta secundária (Guariguata \& Ostertag 2001). Por outro lado, o retorno à composição florística similar ao da floresta madura é um processo mais lento devido, particularmente, ao crescimento mais lento das árvores emergentes (Finegan 1996). Dentro da sequência de eventos que ocorrem após o início do processo de regeneração natural (Guariguata \& Ostertag 2001), a parcela N está na etapa em que a floresta retorna à composição florística similar à floresta mais antiga.

A porção nordeste do PESM é importante do ponto de vista ecológico uma vez que, junto ao Parque Nacional da Serra da Bocaina e da Área de Proteção Ambiental de Cairuçu, ambas no Rio 
de Janeiro, formam um contínuo com cerca de 120 mil ha de MA (Lacerda 2001). Este é importante ponto a ser considerado, já que espécies como Ocotea catharinensis Mez (Lauraceae) e E. edulis estão na lista IUCN como espécies ameaçadas para o estado do Rio de Janeiro e para todo o bioma, respectivamente.

\section{Agradecimentos}

Este trabalho foi financiado pela Fundação de Amparo à Pesquisa do Estado de São Paulo (FAPESP) no âmbito do Projeto Temático Gradiente Funcional: Composição florística, estrutura e funcionamento da Floresta Ombrófila Densa dos Núcleos Picinguaba e Santa Virgínia do Parque Estadual da Serra do Mar (Processo 03/12595-7), que faz parte do Programa BIOTA/FAPESP - O Instituto Virtual da Biodiversidade (www.biota.org.br). Autorização COTEC/IF 41.065/2005 e autorização IBAMA/CGEN 093/2005. Agradecemos também ao Programa de Pós-Graduação em Biologia Vegetal da Universidade Estadual de Campinas, à FAPESP e ao CNPq, respectivamente pelas bolsas de mestrado da primeira e da segunda autora. Finalmente, agradecemos aos taxonomistas do Laboratório de Taxonomia Vegetal da UNICAMP pelo auxílio na identificação das espécies, ao pesquisador Pedro Eisenlohr pela ajuda na análise dos dados, a equipe do Parque Estadual da Serra do Mar/Núcleo Santa Virgínia e ao motorista/auxiliar de campo Renato Belinello pela inestimável ajuda no trabalho de campo.

\section{Referências Bibliográficas}

AGUIRRE, G.H. 2008. Caracterização da vegetação arbustivo-arbórea de fragmentos de floresta ombrófila densa montana. Dissertação de Mestrado, Universidade Estadual de Campinas, Campinas.

ALVES, L.F. \& METZGER, J.P. 2006. A regeneração florestal em áreas de floresta secundária na Reserva Florestal do Morro Grande, Cotia, SP. Biota Neotrop. 6 (2): http://www.biotaneotropica.org.br/v6n2/pt/ abstract?article+bn00406022006 . (ultimo acesso em 27/11/2010).

ANGIOSPERM PHYLOGENY GROUP - APG II. 2003. An update of the Angiosperm Phylogeny Group classification for the orders and families of flowering plants: APG II. Bot. j. Linn. Soc. 141(4):399-436. http:// dx.doi.org/10.1046/j.1095-8339.2003.t01-1-00158.x

AYRES, M., AYRES JUNIOR, M., AYRES, D.L. \& SANTOS, A.A.S. 2007. Biostat (5.0): Aplicações estatísticas nas áreas das ciências biomédicas. Belém-Pará.

AYRES, M., AYRES JUNIOR, M., AYRES, D.L. \& SANTOS, A.A.S. 2007. Biostat (5.0): Aplicações estatísticas nas áreas das ciências biomédicas. Belém-Pará.

BARBOSA, T.D.M. 2009. A família Lauraceae Juss. No município de Santa Teresa, Espírito Santo. Dissertação de Mestrado, Universidade Estadual de Campinas, Campinas.

BERTONCELLO, R. 2009. A vegetação arbórea em um gradiente altitudinal no Morro do Cuscuzeiro, Ubatuba (SP): uma análise florística, fitossociológica e fitogeográfica. Dissertação de Mestrado, Universidade Estadual de Campinas, Campinas.

BREARLEY, FRANCIS Q., SUKAESIH PRAJADINATA, PETRA S. KIDD, JOHN PROCTOR \& SURIANTATA. 2004. Structure and floristics of an old secondary rain forest in Central Kalimantan, Indonesia, and a comparison with adjacent primary forest. Forest Ecology and Management, 195: 385-397.

BROWER, J.E.\& ZAR, J.H. 1984. Field and laboratory methods for general ecology. Wm. C. Brown Pub., Dubuque. http://dx.doi.org/10.1017/ S0266467400003989

BROWN, S. \& LUGO, A.E. 1990. Tropical secondary forests. J. Trop. Ecol 6(1):1-32.

BRUMMITT, R.K. \& POWELL, C.E. 1992. Authors of plant names. Royal Botanic Gardens.
CESCO, S. \& CABRAL, D.C. 2008. Notas para uma história da exploração madeireira na mata atlântica do sul-sudeste. Ambient. Soc. 11:33-48.

COLWELL, R.K. 2009. EstimateS: Statistical estimation of species richness and shared species from samples. Version 8.2. http://viceroy.eeb.uconn. edu/EstimateSPages/EstSUsersGuide/EstimateSUsersGuide.htm (último acesso em 20/10/2010).

COLWELL, R.K., MAO, C.X. \& CHANG, J. 2004. Interpolating, extrapolating, and comparing incidence-based species accumulation curves. Ecology. 85:2717-2727. http://dx.doi.org/10.1890/03-0557

DIAS, A.C. 2005. Composição florística, fitossociologia, diversidade de espécies arbóreas, e comparação de métodos de amostragem na floresta ombrófila densa no Parque Estadual Carlos Botelho/SP - Brasil. Tese de Doutorado, Escola Superior de Agricultura Luiz de Queiros, Universidade de São Paulo, Piracicaba.

DEAN, W. 2002. A ferro e fogo: A história e a devastação da Mata Atlântica brasileira. Companhia das Letras, São Paulo. 484p.

FALKENBERG, D.B. \& VOLTOLINI, J.C. 1993. The Montane Cloud Forestin Southern Brazil. In Tropical Montane Forests (L.S. Hamilton, J.O. Juvik \& F.N. Scatena). East-West Center, Unesco. p.86-93.

FIDALGO, O. \& BONONI, V.L.R. 1984. Técnicas de coleta, preservação e herborização de material botânico. Instituto de Botânica, São Paulo.

FINEGAN, B. 1996. Pattern and process in neotropical secondary rain forests: the first 100 years of succession. Tree. 11(3):119-124. http://dx.doi. org/10.1016/0169-5347(96)81090-1

FONTES, M.A.L. 1997. Análise da composição florística das Florestas Nebulares do Parque Estadual de Ibitipoca, Minas Gerais. Dissertação de Mestrado, Universidade Federal de Lavras, Lavras.

GENTRY, A.H. 1995. Patterns of diversity and floristics composition in a neotropical montane forests. In Neotropical Montane Forests Biodiversity and Conservation Symposium. The New York Botanical Garden, New York.

GHAZOUL, J. \& SHEIL, D. 2010. Tropical Rain Forest: Ecology, Diversity, and Conservation. Oxford University Press, New York.

GOTELLI, N.J. \& COLWELL, R.K. 2001. Quantifying biodiversity: procedures and pitfalls in the measurement and comparison of species richness. Ecol. Lett. 4:379-391. http://dx.doi.org/10.1046/j.14610248.2001.00230.x

GUARIGUATA, M.R. \& OSTERTAG, R. 2001. Neotropical secondary forest succession: changes in structural and functional characteristics. For. Ecol. Manage. 148:185-206. http://dx.doi.org/10.1016/S0378$1127(00) 00535-1$

GUILHERME, F.A.G., MORELLATO, L.P.C. \& ASSIS, M.A. 2004. Horizontal and vertical tree community structure in a lowland Atlantic Rain Forest, Southeastern Brazil. Rev. Bras. Bot. 27:725-737.

HIROTA, M.M. 2003. Monitoring the Brazilian Atlantic Forest cover. In The Atlantic Forest of South America: biodiversity status, trends, and outlook (C. Galindo-Leal \& I.G. Câmara, eds.). Center for Applied Biodiversity Science e Island Press, Washington. p.60-65.

INSTITUTO FLORESTAL - IF. 2010. Unidades de Conservação do Estado de São Paulo. Disponível em: http://www.iflorestal.sp.gov.br/unidades_ conservacao/index.asp. (último acesso em 14/01/2010).

JOLY, C.A., ASSIS, M.A., BERNACCI, L.C., TAMASHIRO, J.Y., CAMPOS, M.C.R., GOMES, J.A.M.A., SANCHEZ, M., SANTOS, F.A.M., PEDRONI, F., PEREIRA, L.S., PADGURSCHI, M.C., PRATA, E.M.B., RAMOS, E., TORRES, R.B., ROCHELLE, A.L.C., MARTINS, F.R, ALVES, L.F., VIEIRA, S.A., MARTINELLI, L.A., CAMARGO, P.B., SIMÕES, E., VILLANI, J.P. \& BELINELLO, R. 2011. Florística e fitossociologia do componente arbóreo da Mata Atlântica ao longo do gradiente altitudinal dos Núcleos Picinguaba e Santa Virgínia/PESM, do sudeste do Brasil. Biota Neotrop. 11(3): no prelo.

JOST, L. 2006. Entropy and diversity. Oikos. 113(2):363-375. http://dx.doi. org/10.1111/j.2006.0030-1299.14714.x 
JOST, L. 2010. New synthesis of diversity indices and similarity measures. Disponível em: http://www.loujost.com/Statistics\%20and\%20Physics/ Diversity\%20and\%20Similarity/DiversitySimilarityHome.htm (último acesso 23/11/2010).

KÖEPPEN, W. 1948. Climatologia. Fondo de Cultura Econômica, México.

KREBS, C.J. 1999. Ecological Methodology. Ed. Adson Wesley Longman Inc., Menlo Park

LACERDA, M.S. 2001. Composição florística e estrutura da comunidade arbórea num gradiente altitudinal da Mata Atlântica. Tese de Doutorado, Universidade Estadual de Campinas, Campinas.

LIEBSCH, D., GOLDENBERG, R. \& MARQUES, M.C.M. 2007. Florística e estrutura de comunidades vegetais em uma cronosequência de Floresta Atlântica no estado do Paraná, Brasil. Acta Bot. Bras. 21(4):983-992. http://dx.doi.org/10.1590/S0102-33062007000400023

MAGURRAN, A.E. 2004. Measuring Biological Diversity. 2nded. Blackwell, Oxford.

MANTOVANI, W. 1993. Estrutura e dinâmica da Floresta Atlântica na Juréia, Iguape, SP. Tese Livre Docente, Universidade de São Paulo, São Paulo.

MAO, C.X. \& COLWELL, R.K. 2005. Estimation of species richness: mixture models, the role of rare species, and inferential challenges. Ecology. 86:1143-1153. http://dx.doi.org/10.1890/04-1078

MARTINS, F.R. 1989. Fitossociologia de florestas do Brasil: um histórico bibliográfico. Pesqui., Bot. 40:103-164.

MARTINS, F.R. 1991. Estrutura de uma floresta mesófila. Editora UNICAMP, Campinas.

MARTINS, S.C. 2010. Caracterização dos solos e serapilheira ao longo do gradiente altitudinal da Mata Atlântica, estado de São Paulo. Tese de Doutorado, Centro de Energia Nuclear na Agricultura, Universidade de São Paulo, Piracicaba.

MORI, S.A., MATTOS-SILVA, L. A., LISBOA, G. \& CORADIN, L. 1989. Manual de manejo do herbário fanerogâmico. 2nd ed. CEPLAC, Ilhéus.

MÜELLER-DOMBOIS, D. \& ELLENBERG, H. 1974. Aims and methods for vegetation ecology. J. Wiley \& Sons, New York.

MYERS, N., MITTERMEIER, R.A., MITTERMEIER, C.G., FONSECA, G.A.B., KENT, J. 2000. Biodiversity hotspots for conservation priorities. Nature. 403:853-858. http://dx.doi.org/10.1038/35002501

OLIVEIRA-FILHO, A.T. \& FONTES, M.A.L. 2000. Patterns of floristic differentiation among Atlantic Forests in Southeastern Brazil, and the influence of Climate. Biotropica. 32:793-810.
OLIVEIRA-FILHO, A.T., VILELA, E.A., GAVILANES, M.L. \& CARVALHO, D.A. 1994. Effect of flooding regime and understorey bamboo on the physiognomy and tree species composition of a tropical semideciduous forest in the southeastern Brazil. Vegetatio. 113:99-124.

PADGURSCHI, M.C.G. 2010. Composição e estrutura arbórea de um trecho de Floresta Ombrófila Densa Montana com taquaras na Mata Atlântica. Dissertação de Mestrado, Universidade Estadual de Campinas, Campinas.

PEIXOTO, A.L. 1987. Revisão taxonômica do gênero Mollinedia Ruiz et Pavon (Monimiaceae, Monimioideae). Tese de Doutorado, Universidade Estadual de Campinas, Campinas.

RIBEIRO, M.C., METZGER, J.P., MARTENSEN, A.C., PONZONI, F.J. \& HIROTA, M.M. 2009. The Brazilian Atlantic Forest: How much is left, and how is the remaining forest distributed? Implications for conservation. Biol. Conserv. 142:1141-1153. http://dx.doi.org/10.1016/j. biocon.2009.02.021

RICHARD, P.W. 1996. The tropical rain Forest an ecological study. 2nd ed. Cambridge University Press, Cambridge.

ROBIM, M.J., PASTORE, J.A., AGUIAR, O.T. \& BAITELLO, J.B. 1990. Flora arbóreo-arbustiva e herbácea do Parque Estadual de Campos do Jordão (SP). Rev. IF. 2(1):31-53.

SHEPHERD, G.J. 2008. Fitopac 2.0: manual do usuário. Departamento de Botânica/UNICAMP, Campinas.

TABARELLI, M. \& MANTOVANI, W. 2000. Gap-phase regeneration in a tropical montane forest: the effects of gap structure and bamboo species. Plant Ecol. 148:149-155. http://dx.doi.org/10.1023/A:1009823510688

TABARELLI, M., PINTO, L.P., SILVA, J.M.C., HIROTA, M.M. \& BEDÊ, L.C. 2005. Desafios e oportunidades para a conservação da biodiversidade na Mata Atlântica brasileira. Megadiversidade. 1(1):132-138.

TABARELLI, M., VILLANI, J.P. \& MANTOVANI, W. 1993. Aspectos da sucessão secundária em trecho da floresta atlântica no parque Estadual da Serra do mar, SP. Rev. IF. 5(1):99-112.

TABARELLI, M., VILLANI, J.P. \& MANTOVANI, W. 1994. Estudo comparativo da vegetação de dois trechos de floresta secundária no Núcleo Santa Virgínia/SP. Rev. IF. 6(1):1-11.

TONHASCA JÚNIOR, A. 2005. Ecologia e história natural da Mata Atlântica. Editora Interciência.

VELOSO, H. P., RANGEL FILHO, A. L. R. \& LIMA, J. C. A. 1991. Classificação da Vegetação Brasileira, Adaptada a um Sistema Universal. IBGE, Departamento de Recursos Naturais e Estudos Ambientais, Rio de Janeiro.

ZAR, J.H. 1999. Bioestatistical Analysis. 4nd ed. Prentice-Hall Inc., New Jersey. 